\title{
BET Bromodomain Inhibitors Target the NEUROD1-subtype SCLC by Blocking NEUROD1 Transactivation
}
Haobin Chen ${ }^{1, \#}$, Lisa Gesumaria ${ }^{1}$, Young-Kwon Park ${ }^{2}$, Trudy G. Oliver ${ }^{3}$, Dinah S. Singer ${ }^{4,}$, Kai $\mathrm{Ge}^{2, *}$, David S. Shrump ${ }^{1, *}$

1 Thoracic Surgery Branch, Center for Cancer Research, National Cancer Institute, National Institutes of Health, Bethesda, MD 20892, USA

${ }^{2}$ Adipocyte Biology and Gene Regulation Section, Laboratory of Endocrinology and Receptor Biology, National Institute of Diabetes and Digestive and Kidney Diseases, National Institutes of Health, Bethesda, MD 20892, USA

${ }^{3}$ Department of Oncological Sciences, Huntsman Cancer Institute, University of Utah, Salt Lake City, UT 84112, USA

${ }^{4}$ Experimental Immunology Branch, Center for Cancer Research, National Cancer Institute, National Institutes of Health, Bethesda, MD 20892, USA

* Senior authors

\section{\# Corresponding author}

Address: 10 Center Drive Room 3-5888, Thoracic Surgery Branch, National Cancer Institute, National Institutes of Health, Bethesda, MD 20892, USA

Tel: 240-760-6177

Email: haobin.chen@,nih.gov

Funding: This study was supported by the Center for Cancer Research, the Intramural Program of the National Cancer Institute (NCI) (grant ZIA BC011787: Chen), and in part through a FLEX award from the Center for Cancer Research, NCI (grant ZIA BC011839: Chen and Schrump) and NCI U01 CA231844 (Oliver).

Conflict of Interest: The authors declare no conflict of interest. 


\begin{abstract}
SCLC is a recalcitrant malignancy with a dismal prognosis. Four molecular subtypes of SCLC have been named, each associated with one master transcription factor (ASCL1, NEUROD1, POU2F3, or YAP1). Much is still unknown about NEUROD1's function and transactivation in this malignancy. Herein we report that knockout of NEUROD1 triggered SCLC to evolve into a YAP1 subtype. Through an integrated analysis of RNA-seq and ChIP-seq, we found NEUROD1 regulates neural-related genes by binding to gene promoters and distal enhancers. NEUROD1 physically interacts with BET bromodomain proteins and recruits them to actively transcribed genes. Inhibition of BET bromodomain proteins blocks NEUROD1 transactivation and suppresses SCLC growth. We identified LSAMP as one of the NEUROD1-target genes that mediate SCLC's sensitivity to BET bromodomain inhibitors. Our findings suggest that targeting transcriptional coactivators could be a new approach to block master transcription factors in SCLC to enable precision therapy.
\end{abstract}

Keywords: Molecular Subtype Evolution; BRD4; LSAMP; Epigenetic; Superenhancer; Biomarker 


\section{INTRODUCTION}

Small cell lung cancer (SCLC) is a recalcitrant malignancy [1]. Despite recent advances in therapeutics, durable responses are typically infrequent and its prognosis remains dismal [2, 3]. Although SCLC has been treated as a single disease, recent evidence reveals that it is more heterogeneous. A newly proposed classification divided it into four molecular subtypes (ASCL1, NEUROD1, POU2F3, and YAP1), with each subtype named after one master transcription factor identified in SCLC cell lines $[4,5]$. There is emerging evidence that various molecular subtypes of SCLC have different therapeutic susceptibilities, likely because of their distinct transcriptional landscapes [6]. Thus, studying gene regulation in each molecular subtype of SCLC would likely inform the development of subtype-specific therapies, which may improve the outcomes of this devastating cancer.

NEUROD1 is a neurogenic basic helix-loop-helix transcription factor and plays a critical role in neuronal differentiation [7]. Previous studies in embryonic stem cells and microglia cells found that NEUROD1 could induce the neuronal genes by directly binding to target heterochromatic promoters and enhancers to initiate chromatin and transcription factor reprogramming $[8,9]$. NEUROD1 is critical for cell survival in a subset of SCLC lines, and its knockdown decreased cell migration and invasion in part through an NTRK2- (previously known as TrkB) and NCAM-dependent mechanism [10]. How NEUROD1 regulates gene expression is less clear in SCLC. A previous study has established that NEUROD1 and ASCL1 largely bind to different genomic regions in SCLC [11]. However, due to a lack of transcriptome data in the same cell lines with perturbed NEUROD1 expression (e.g., overexpression or knockout), it is uncertain what genes are directly regulated by and to what degree these genes are dependent on NEUROD1. In addition, how transcription cofactors facilitate the transactivation of NEUROD1 has not been 
characterized in SCLC. Currently, there is no subtype-specific therapy for SCLC in the clinic. Although Seneca Valley virus was found to preferably infect the variant subtype of SCLCs that commonly express NEUROD1 [12], no anti-tumor activity was found in a phase II trial of nonselective patients with relapsed SCLC [13].

The bromodomain and extraterminal domain (BET) family of proteins, including BRD2, BRD3, BRD4, and BRDT, are involved in multiple aspects of transcriptional regulation [14]. Two bromodomains at the N-terminus of this family of proteins are critical for binding acetylated histone lysines on active chromatin $[14,15]$. BET bromodomain inhibitor (BETi) selectively binds to these bromodomains. As a result, BET bromodomain proteins cannot access active chromatin, resulting in gene transcription inhibition [16, 17]. Although BET bromodomain proteins are broadly associated with gene promoters and enhancers, BETi only inhibits a subset of genes, particularly those related to cell lineage and driver oncogenes [15]. In diffuse large B-cell lymphoma and multiple myeloma, the genes susceptible to BETi had unusually high levels of BRD4 occupancy at their enhancer sites, commonly known as superenhancers (SEs) [18, 19].

In this study, we investigated how NEUROD1 inactivation affects gene regulation and how NEUROD1 could be targeted for therapeutic purposes in SCLC.

\section{RESULTS}

\section{Knockout (KO) of NEUROD1 triggers molecular subtype evolution in SCLC.}

To study the function of NEUROD1 in SCLC, we knocked out this gene in H446 cells using CRISPR. Western blot and Sanger sequencing were performed for verification (Fig. 1A-B). A drastic decrease in the expression of NEUROD2, a known target gene of NEUROD1, functionally confirmed disruption of the NEUROD1 network in the H446 NEUROD1-KO clones 
(Fig. 1C). We observed that the appearance of the H446 NEUROD1-KO cells was remarkably different from control cells. As shown in Figure 1D, the H446 NEUROD1-KO cells (bottom three) had an elongated shape and a monolayered growth pattern, while control cells (top three) were round and clumpy in appearance. We found similar changes in cell appearance when we knocked out NEUROD1 using a different guide RNA (gRNA) in H446 cells, indicating this was not due to an off-target effect of CRISPR (Fig. S1A-B). We next tested the growth of the NEUROD1-KO cells in vitro. As shown in Figure 1E, the H446 NEUROD1-KO cells grew slower in vitro compared to control cells. Furthermore, these NEUROD1-KO cells formed fewer and smaller xenografts in immunodeficient mice, despite several additional weeks of growth (Fig. 1F). These results suggest that NEUROD1 is critical for SCLC tumorigenicity and proliferation.

To identify the genes involved in these changes upon NEUROD1 KO, we profiled three H446 KO clones and three control clones by RNA sequencing (RNA-seq; Table S2). Gene Set Enrichment Analysis (GSEA) showed that, in the H446 NEUROD1-KO cells, the neuroendocrine (NE) gene signature was depleted while the epithelial-mesenchymal transition (EMT) and YAP1 signatures were enriched (Fig. 1G). We validated these results using quantitative reverse transcription-PCR (qRT-PCR) in the H446 NEUROD1-KO cells generated using two different gRNAs. As expected, CHGA and INSM1, two neuroendocrine genes, were suppressed in the H446 NEUROD1-KO cells (Fig. 1H). In contrast, CD44 (an EMT gene), YAP1, and CTGF (a YAPtarget gene) were upregulated, suggesting activation of EMT and YAP1 signaling after the KO of NEUROD1 (Fig. 1H). Consistent with a recent study that found Notch signaling critical for SCLC molecular subtype evolution [20], the H446 NEUROD1-KO cells had a significant increase in expression of NOTCH1 and its target genes, HESI and REST (Fig. 1H). We confirmed YAP1 protein was induced upon the KO of NEUROD1 in H446 cells (Fig. 1I). Furthermore, when YAP1 
bioRxiv preprint doi: https://doi.org/10.1101/2021.10.25.465771; this version posted October 25, 2021. The copyright holder for this preprint (which was not certified by peer review) is the author/funder. This article is a US Government work. It is not subject to copyright under 17 USC 105 and is also made available for use under a CCO license.

was depleted using two different siRNAs in the H446 NEUROD1-KO cells, a significant decrease in cell viability was observed, suggesting that YAP1 was essential for these cells (Fig. 1J-K).

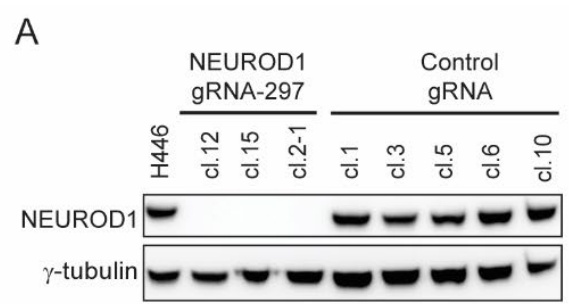

D
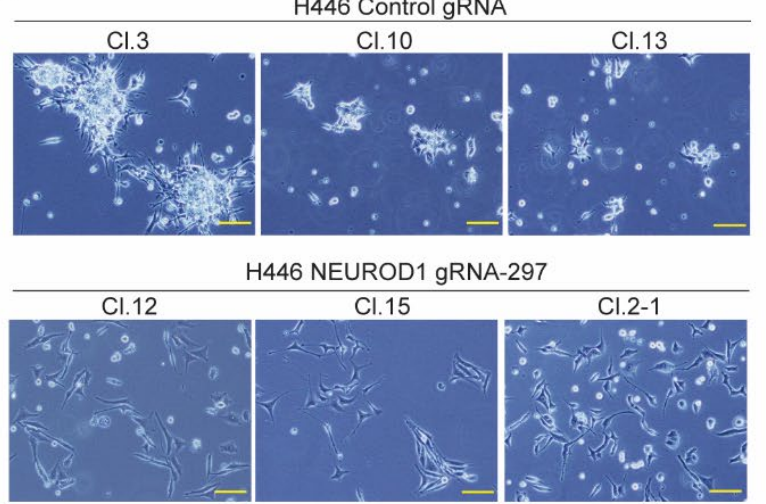

B

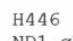

ND1 TCCTCCTCTGCGT-TCATGGTTCGAGGTCGT ND1 gRNA-297 cl.15 TCCTCCTC-------ATGGTTTCGAGGTCGT ND1 gRNA-297 cl.2-1 TCCTCCTCTGCGTTTCATGGTTTCNAGGTCGT

$\mathrm{F}$
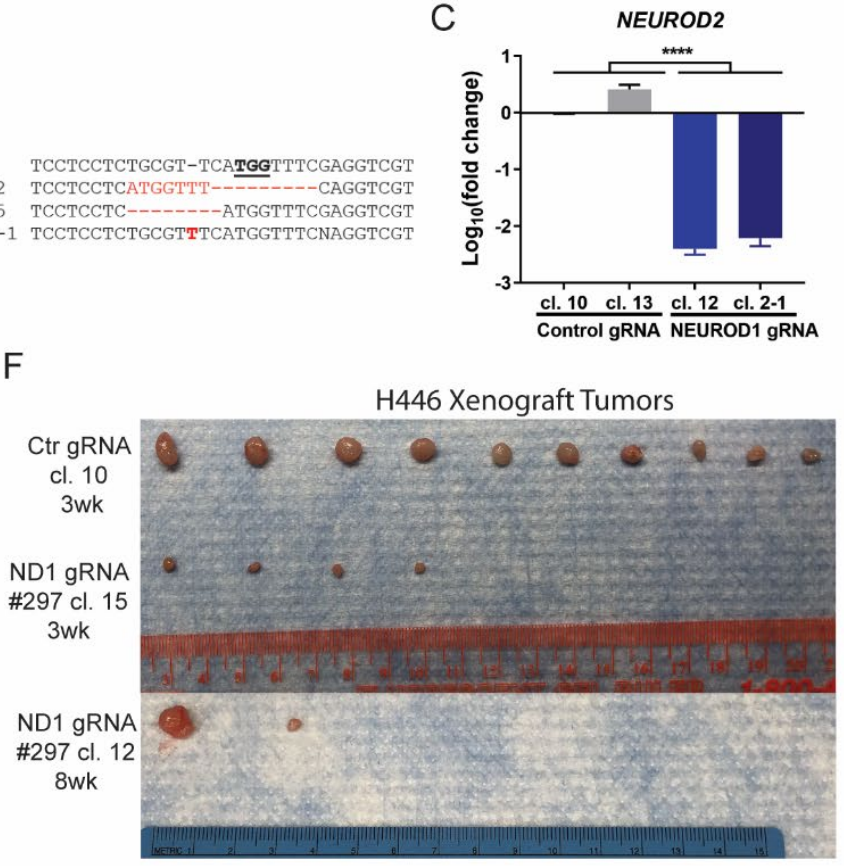

E

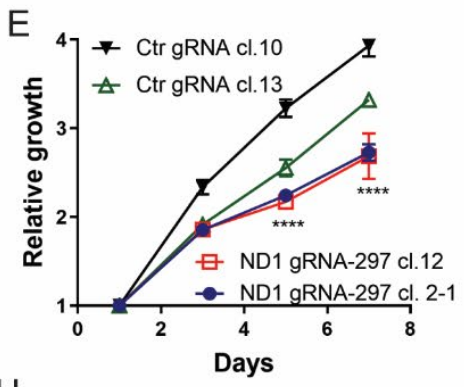

$\mathrm{H}$

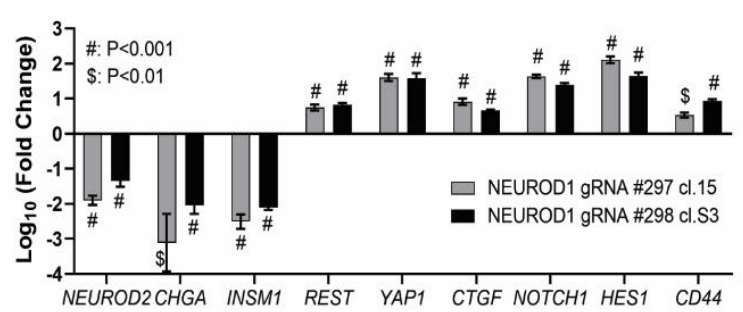

G

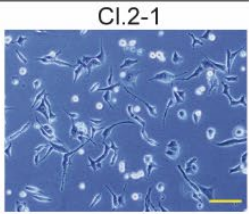

C
Neuroendocrine gene signature Hallmark of EMT

YAP1 Conserved signature
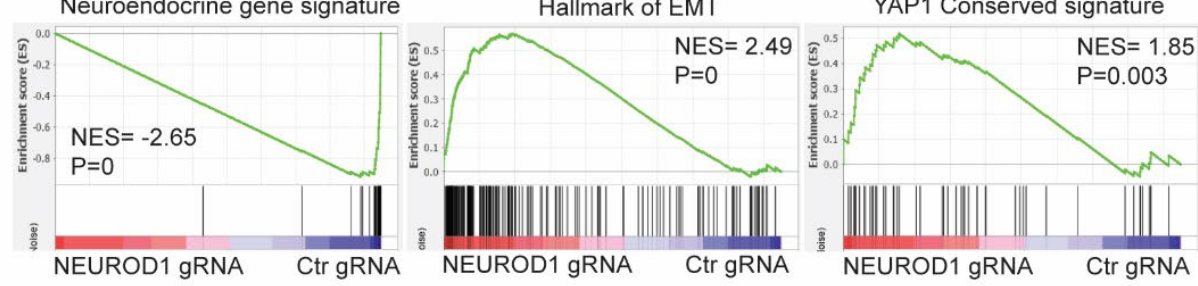

I

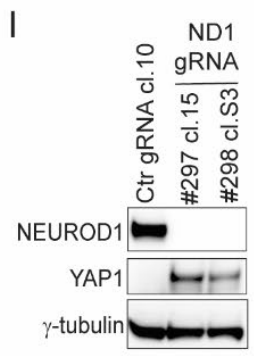

$\mathrm{J}$

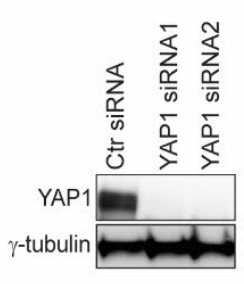

K

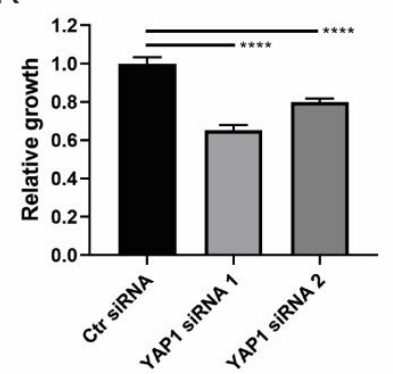

Figure 1. Inactivation of NEUROD1 triggers molecular subtype evolution in H446 cells. A)

Western blots showing the loss of NEUROD1 expression in three H446 NEUROD1-KO clones (gRNA \#297) versus five control clones and parental H446 cells. B) The aligned Sanger sequencing results showing the indels at the targeted NEUROD1 genomic sequence 
(complementary strand) in three H446 NEUROD1-KO clones. The protospacer adjacent motif (PAM) sequence was underlined, and the indel sequences were highlighted red. C) A substantial decrease of NEUROD2 expression in the H446 NEUROD1-KO clones, as measured by qRT-PCR. D) Distinct appearance of three H446 NEUROD1-KO clones compared to control cell clones. Scale bar: $100 \mu \mathrm{m}$. E) Comparison of cell proliferation between the H446 NEUROD1-KO clones and control cell clones. F) Impaired xenograft formation of the H446 NEUROD1-KO cells (\#297 cl.15 and cl.12) compared to control cells (Ctr gRNA cl.10). $\mathrm{n}=10$ per clone. Top, three weeks after inoculation. Bottom, eight weeks after injection. G) GSEA plots of neuroendocrine signature, epithelial to mesenchymal transition (EMT) signature, and YAP1 conserved gene signature comparing H446 NEUROD1-KO cells (pooled data of \#297 cl. 12, cl. 15, and cl. 2-1) to control cells (pooled data of cl. 3, cl. 10, and cl. 13). H) Relative changes in the expression of select genes ( $\log _{10}$ [Fold Change]), as measured by qRT-PCR, comparing H446 NEUROD1-KO (\#297 cl. 15 or \#298 cl. S3) to control cells (cl. 10). I) Western blots showing YAP1 protein expression in the H446 NEUROD1-KO cells (\#297 cl. 15 and \#298 cl. S3). J) Western blots showing knockdown of YAP1 protein in the H446 NEUROD1-KO cells (\#297 cl. 12) using two different siRNAs. K) Decreased cell proliferation after knocking down YAP1 in H446 NEUROD1-KO cells (\#297 cl. 12). The significance of two-group comparisons was determined using the Student's $t$-test in (C), $(\mathrm{E})$, and $(\mathrm{H})$ and the ANOVA test with multi-comparison correction in $(\mathrm{K})$. When indicated, ****, $\mathrm{P}<0.0001$. Error bars represent SD. Ctr, control; gRNA, guide RNA; ND1, NEUROD1; cl., clone; NES, normalized enrichment score; siRNA, small interfering RNA.

We knocked out NEUROD1 in another NEUROD1-subtype SCLC line, DMS-273, to validate the above findings in H446 cells. Like H446, the KO of NEUROD1 induced YAP1 expression in DMS-273 (Fig. S2A). We compared gene expression changes between H446 and DMS-273 upon the KO of NEUROD1 (Fig. S2B). Both NEUROD1-KO cells showed similar gene expression changes related to the NE, EMT, and YAP1 signaling, but the NOTCH signaling genes remained unaffected in the DMS-273 NEUROD1-KO cells. Collectively, these findings demonstrate that NEUROD1 KO triggers molecular subtype evolution in multiple SCLC lines. 


\section{NEUROD1 regulates neural-related genes by binding to both promoters and distal} enhancers.

To understand how NEUROD1 KO caused these changes in gene expression, we first performed chromatin immunoprecipitation with massively parallel sequencing (ChIP-seq) to map NEUROD1 genome-wide occupancy in H446 control cells (Table S3). As summarized in Figure 2A, most of the NEUROD1 peaks $(\mathrm{N}=68,395)$ located in intronic $(45.8 \%)$ and intergenic regions (45.9\%), with only small percentages present at transcription start sites (TSS; $1.12 \%)$, promoterTSS sites (3.88\%), and exons +3 ' or 5' untranslated regions (UTRs; 3.28\%). Using a de novo motif analysis, we found NEUROD1/NEUROG2 binding motifs were enriched in the corresponding genomic sequences of the identified NEUROD1 peaks (Fig. S3).

A

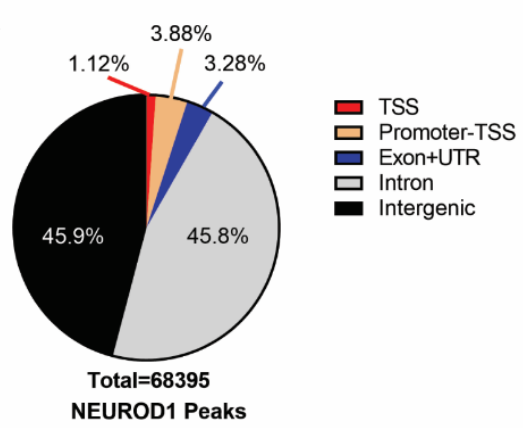

B

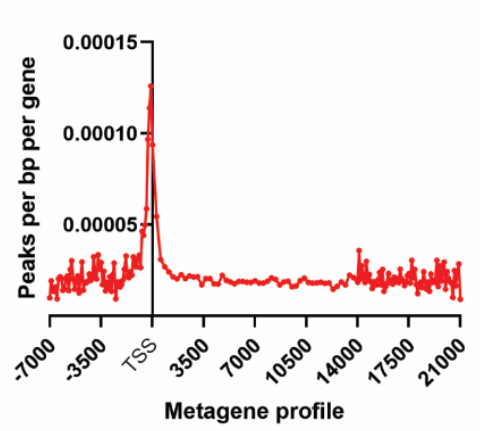

$\mathrm{E}$

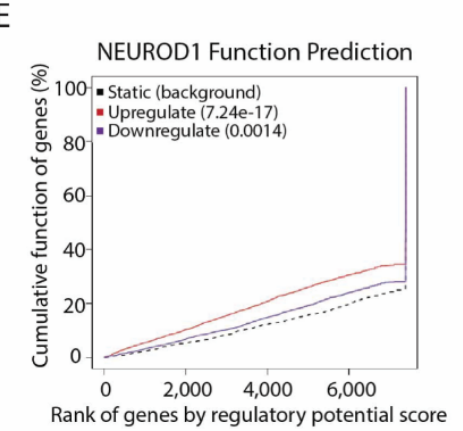

$\mathrm{F}$
C
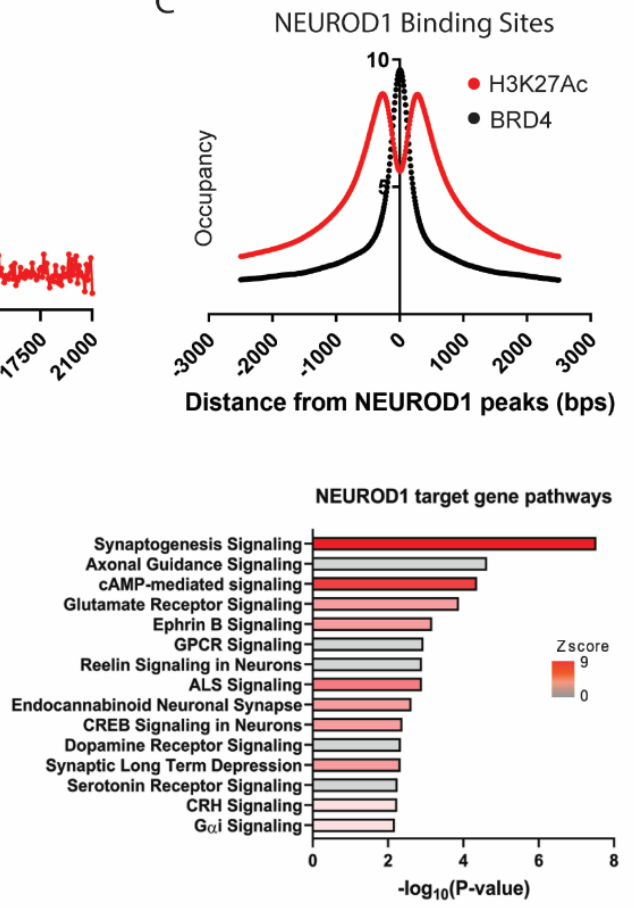

Figure 2. NEUROD1 regulates gene expression at both promoter and distant enhancers. A) A pie chart showing the breakdown of the genomic locations of NEUROD1 peaks in H446 control cells. B) A metagene profile of NEUROD1 peaks across the transcriptome of H446 control cells. C) Genome-wide histone H3 lysine 27 acetylation (H3K27Ac) and BRD4 occupancy within 2.5 
kilobases of the NEUROD1 peaks in the H446 control cells. D) A flow chart illustrating the identification of NEUROD1-target genes $(\mathrm{N}=1,216)$ through an integrated analysis of the RNAseq (H446 NEUROD1-KO cells vs. control cells) and ChIP-Seq data (H446 control cells). E) The transcriptional activity of NEUROD1 predicted by the Binding and Expression Target Analysis algorithm. F) Ingenuity Pathway Analysis identified multiple neural-related pathways enriched in the NEUROD1-target genes. TSS, transcription start site; UTR, untranslated region; GEPs, gene expression profiles; GPCR, G protein-coupled receptor; ALS, amyotrophic lateral sclerosis; CRH, corticotropin-releasing hormone.

We next performed a metagene analysis to assess the genomic locations of the NEUROD1 peaks in relation to the transcription start sites (TSSs). As shown in Figure 2B, NEUROD1 occupancy peaked at the TSS sites, but it was also present within 7KB upstream or at the distal downstream regions $(>14 \mathrm{~KB})$, indicating that NEUROD1 also binds to distal regulatory elements in the genome. To examine this possibility, we evaluated its association with histone H3 lysine 27 acetylation (H3K27Ac; a histone marker for active enhancers) and BRD4 (a marker for active enhancers and promoters). As shown in Figure 2C, BRD4 occupancy peaked at the center of NEUROD1 peaks, while H3K27Ac occupancy was in a typical peak-valley-peak pattern surrounding NEUROD1 peaks, suggesting that NEUROD1 binds to both promoters and enhancers.

To identify NEUROD1-target genes, we performed an integrated analysis of the ChIP-seq and RNA-seq data. Using the Binding and Expression Target Analysis (BETA) algorithm [21], we identified a total of 1,216 NEUROD1-target genes in H446 control cells (Fig. 2D and Table S4). As predicted by the BETA algorithm, NEUROD1 mainly functioned as a transcriptional activator (Fig. 2E, the red curve). Consistent with the role of NEUROD1 in neuroendocrine differentiation, Ingenuity Pathway Analysis identified enrichment of multiple neural-related pathways among the NEUROD1-target genes (Fig. 2F). Taken together, these findings suggest that NEUROD1 regulates neural-related genes by binding to both promoters and distal enhancers. 


\section{NEUROD1 recruits BET bromodomain proteins to actively transcribed genes.}

Visual inspection of the ChIP-seq results revealed loss of H3K27Ac, BET bromodomain proteins, and RPII occupancy along with the NEUROD1 peaks in the NEUROD1-KO cells (Fig. 3A). We confirmed these changes at the NEUROD2 locus using ChIP-quantitative PCR (Fig. S4). A genome-wide analysis confirmed a substantial loss of H3K27Ac and BET bromodomain proteins occupancy around NEUROD1 peaks (Fig. 3B). To assess if the above changes were due to a genome-wide loss of H3K27Ac occupancy, we extracted the genome positions of several other transcription factors reported in the literature (see the Materials and Methods). Consistent with the transcriptome results in Fig. 1G-H, the KO of NEUROD1 increased H3K27Ac occupancy at the genomic positions occupied by TEAD1 and TEAD4 (two YAP1-signaling transcription factors), ZEB1 (a transcriptional mediator of EMT), and RBPJ (a NOTCH-signaling transcription factor) (Fig. 3C).

To assess whether the KO of NEUROD1 directly led to the decrease in BRD4 occupancy at the genomic positions of NEUROD1 peaks, we separated all BRD4 peaks of the H446 control cells into two groups based on whether they overlayed with the NEUROD1 peaks (Fig. 3D heatmap 1 vs. 4; Table S5-6). At the genomic regions with an overlay of BRD4 and NEUROD1 peaks, the KO of NEUROD1 abrogated the BRD4 occupancy (Fig. 3D heatmap 3 vs. 2); in contrast, at the genomic regions with no NEUROD1 occupancy, the BRD4 occupancy was minimally affected (Fig. 3D heatmap 6 vs. 5). These results suggest that NEUROD1 occupancy defines the BRD4 landscape in the NEUROD1-subtype SCLC. 
A
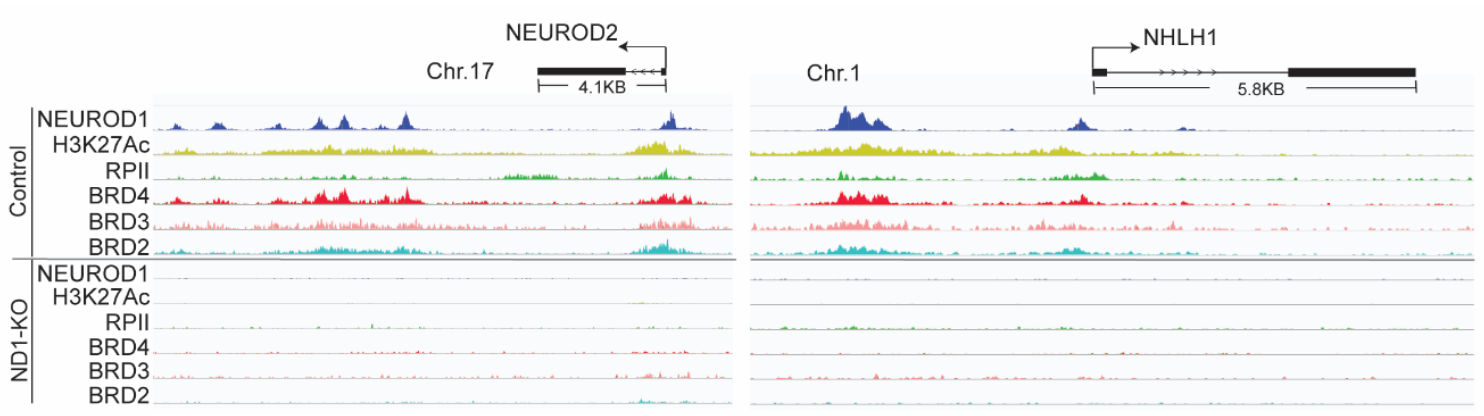

B
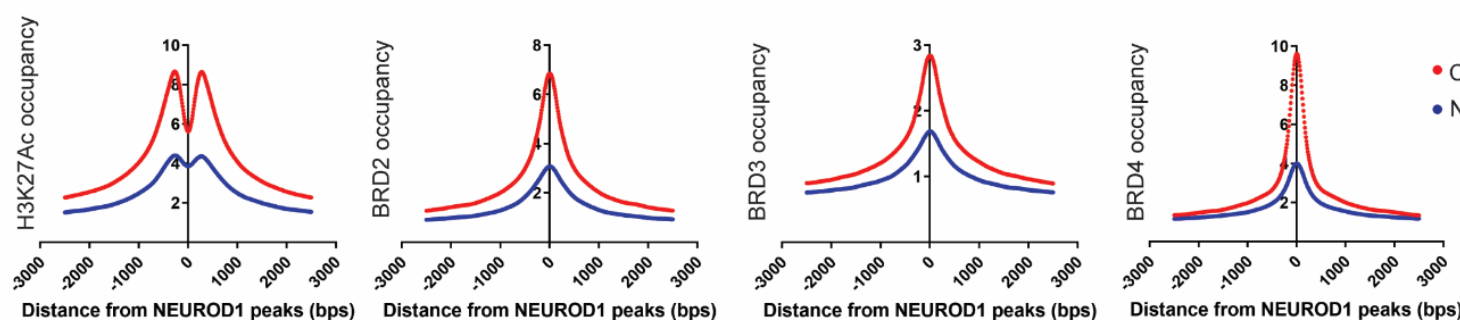

C
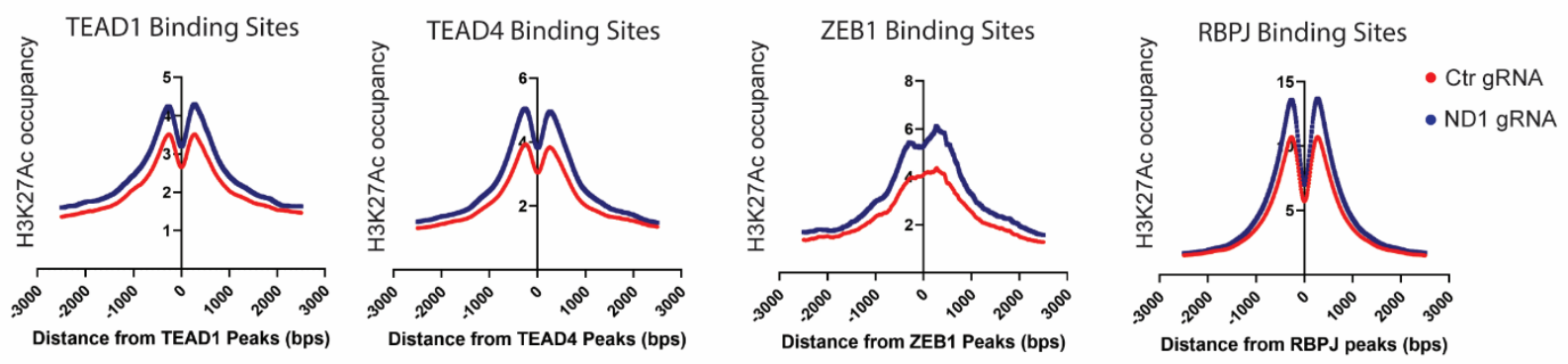

D
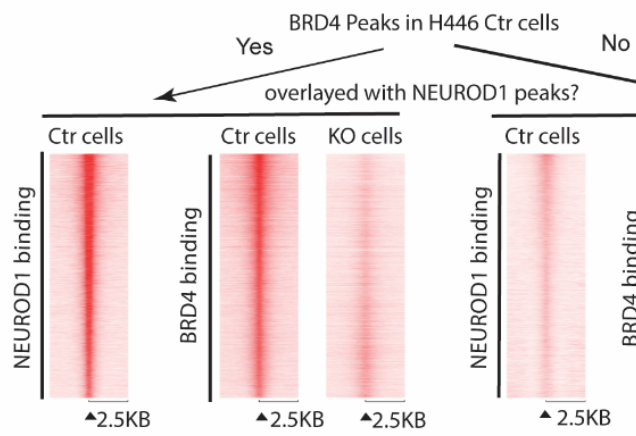

E

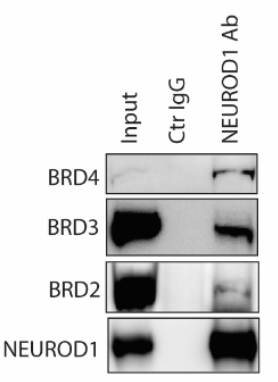

Heatmap \# 1
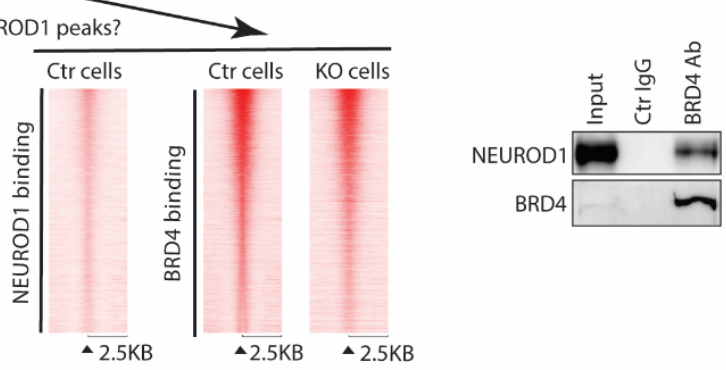

Figure 3. NEUROD1 recruits BET bromodomain proteins to actively transcribed genes. A) Integrated Genomics Viewer (IGV) snapshots showing occupancy of NEUROD1, H3K27Ac, RNA polymerase II (RPII), BRD4, BRD3, and BRD2 at the NEUROD2 (left) and NHLH1 (right) gene loci in the H446 NEUROD1-KO cells (\#297 cl.2-1) versus control cells (Ctr gRNA cl.10). B) Genome-wide H3K27Ac, BRD2, BRD3, and BRD4 occupancy within 2.5 kilobases bilateral of the NEUROD1 peaks in the H446 NEUROD1-KO (\#297 cl.2-1) versus control cells (Ctr gRNA cl.10). C) Genome-wide H3K27Ac occupancy in the H446 NEUROD1-KO cells (\#297 cl.2-1) 
versus control cells (Ctr gRNA cl.10), within 2.5 kilobases bilateral of the genomic positions of TEAD1, TEAD4, ZEB1, and RBPJ peaks extracted from the literature. D) The KO of NEUROD1 affected only a subset of BRD4 peaks that overlayed with NEUROD1 peaks. BRD4 peaks identified in H446 control cells were divided into two groups based on whether they overlayed with NEUROD1 peaks (heatmap 4 vs. 1). The BRD4 peaks overlaying with NEUROD1 peaks in control cells were abrogated upon NEUROD1 KO (heatmap 3 vs. 2), while those not overlaying with NEUROD1 were minimally affected (heatmap 6 vs. 5). E) Co-IP assays using H446 nuclear extracts. Left, immunoblotting for NEUROD1 after IP of BRD4. Right panel: immunoblotting for BRD2, BRD3, and BRD4 after IP of NEUROD1. Ab, antibody; Ctr, control; KO, knockout; ND1, NEUROD1; IP, immunoprecipitation.

To examine whether NEUROD1 physically interacts with BET bromodomain proteins, we performed co-immunoprecipitation (co-IP) assays using the nuclear extracts of H446 and H524 cells. Figures $3 \mathrm{E}$ and S5 show that BET bromodomain proteins co-immunoprecipitated with NEUROD1 and vice versa in both cell lines. Collectively, these findings suggest that NEUROD1 recruits BET bromodomain proteins to actively transcribed genes in SCLC.

\section{BETi suppresses the NEUROD1 gene network.}

The above findings suggest that BET bromodomain proteins function as transcriptional coactivators of NEUROD1. We hypothesized that blocking BET bromodomain proteins would suppress the transcriptional activity of NEUROD1 (Fig. 4A). To test this, we created a NEUROD1 gene signature using 384 NEUROD1-target genes that had at least 4-fold change in gene expression upon the KO of NEUROD1 in H446 cells (Table S7). As shown in Figure 4B, this gene signature was significantly enriched in the NEUROD1-subtype SCLC lines versus all other SCLC lines in the CCLE dataset. JQ1 (a BETi) significantly depleted this NEUROD1 gene signature in 
H446 and COR-L279 cells, consistent with our hypothesis that BETi suppresses the NEUROD1 gene network (Fig. 4C-D, Table S8-10).

A

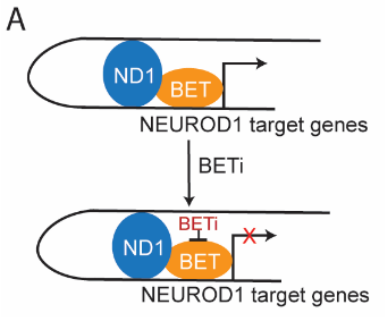

E

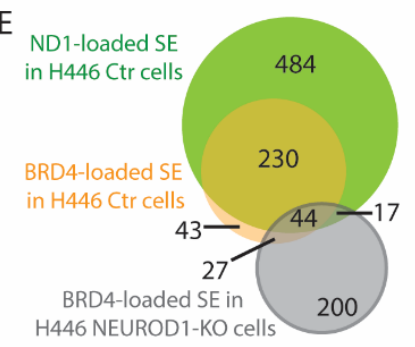

H

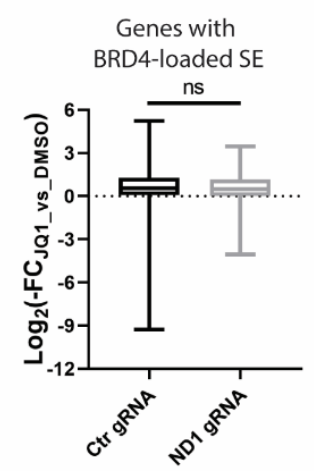

B

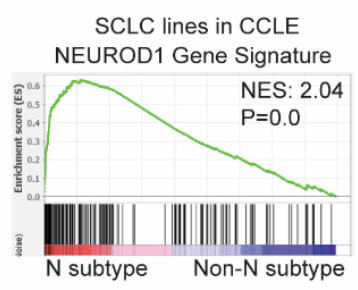

F

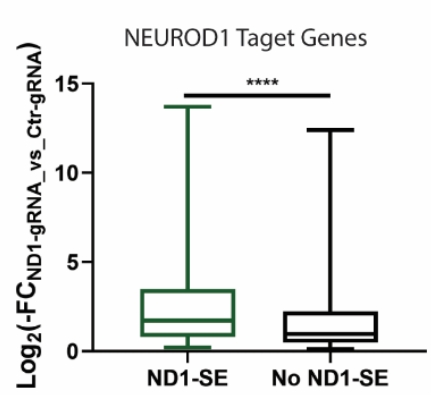

I

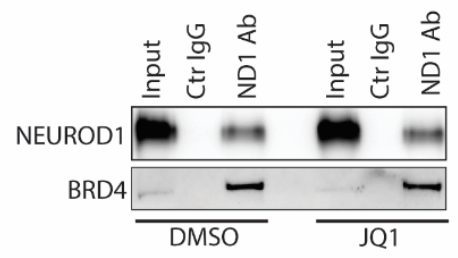

C

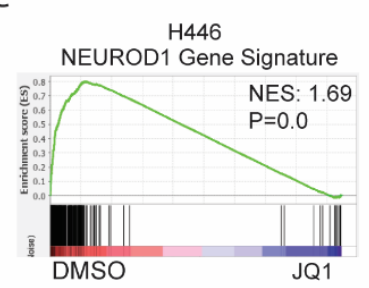

D

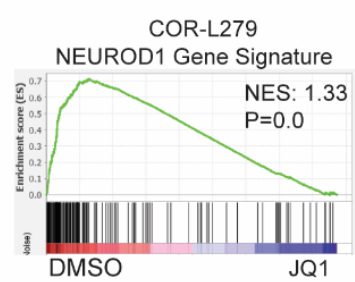

G
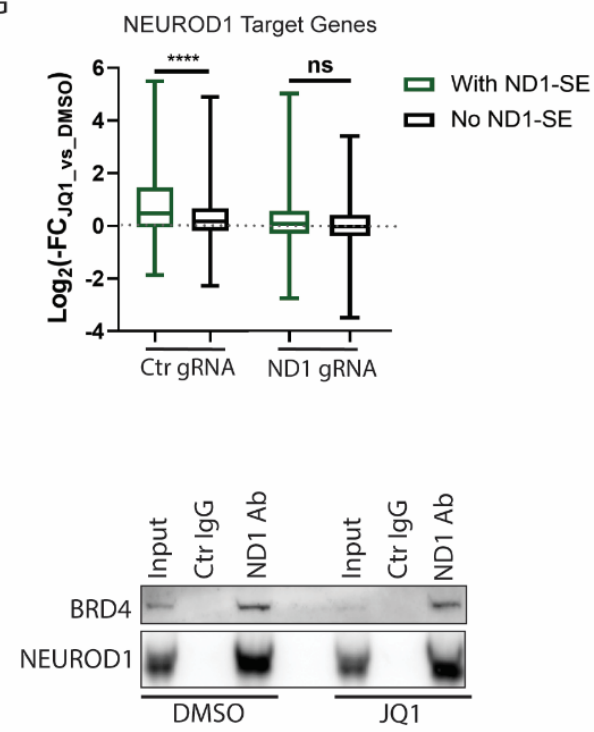

Figure 4. BETi suppresses the NEUROD1 gene network. A) A working model showing that BET bromodomain proteins serve as the transcriptional coactivators of NEUROD1, and BETi could suppress NEUROD1 transactivation by blocking the former proteins. B) GSEA plot of the NEUROD1 gene signature comparing the NEUROD1-subtype SCLC lines (N subtype) to all other SCLC lines (non-N subtype) in the CCLE dataset. C) GSEA plot of NEUROD1 gene signature comparing the JQ1-treated H446 cells ( $1 \mu \mathrm{M} ; 24 \mathrm{hrs})$ to control cells treated with DMSO. D) GSEA plot of NEUROD1 gene signature comparing the JQ1-treated COR-L279 cells $(0.5 \mu \mathrm{M} ; 24$ hrs) to control cells treated with DMSO. E) A Venn diagram showing overlaps among three superenhancer (SE) groups. F) Among NEUROD1-target genes, the genes with the NEUROD1- 
loaded SEs in H446 cells were more significantly suppressed by NEUROD1 KO than those without. G) JQ1 $(1 \mu \mathrm{M}, 24 \mathrm{hrs})$ differentially affected the expression of the NEUROD1-target genes with or without the NEUROD1-loaded SEs in H446 control cells (left two columns) but not in the NEUROD1-KO cells (right two columns). H) JQ1 treatment ( $1 \mu \mathrm{M}, 24 \mathrm{hrs})$ caused a similar degree of suppression in the genes with the BRD4-loaded SEs in the NEUROD1-KO cells as in control cells. I) Co-IP using the nuclear extracts from H446 cells treated with JQ1 (1 $\mu \mathrm{M}, 6 \mathrm{hrs})$ versus DMSO. Left, detection of NEUROD1 by western blot after IP of BRD4. Right, detection of BRD4 after IP of NEUROD1. The significance of the two-group comparisons was determined using the Student's $t$-test in $(\mathrm{F}-\mathrm{H})$. The whiskers in $(\mathrm{F}-\mathrm{H})$ represent minimum to maximum, and the boxes show the first quartile, median, and third quartile. ****, $\mathrm{P}<0.0001$; $\mathrm{Ab}$, antibody; FC, fold change; ND1, NEUROD1; NES, normalized enrichment score; ns, not significant.

To determine whether BETi affects the genes with SEs in SCLC, we first used the H3K27Ac occupancy to mark constituent enhancer sites. We then identified BRD4- or NEUROD1-loaded SEs at least one magnitude larger than the remaining peaks in the enhancer sites (Fig. S6 and Table S11-13). As shown in the Venn diagram of Figure 4E, most NEUROD1loaded SEs intersected with the BRD4-loaded SEs in the H446 control cells, but only a few were in common with the BRD4-loaded SEs in the H446 NEUROD1-KO cells. Among the NEUROD1target genes in H446, those with NEUROD1-loaded SEs were suppressed more significantly by NEUROD1 inactivation than those without (Fig. 4F). Similarly, JQ1 decreased the genes with NEUROD1-loaded SEs more substantially than those without in the H446 control cells (Fig. 4G, left two). In contrast, in the NEUROD1-KO cells, neither of these two lists of genes was affected by JQ1 (Fig. 4G, right two). On the other hand, the genes with BRD4-loaded SEs were suppressed by JQ1 to a similar degree in the H446 NEUROD1-KO cells as in the control cells, indicating that BETi blocked BET bromodomain proteins in both cells (Fig. 4H). 
We next asked whether BETi inhibits NEUROD1 transactivation in part by disrupting the interaction between BET bromodomain proteins and NEUROD1. To examine this possibility, we performed the co-IP assay after treating H446 cells with JQ1. As shown in Figure 4I, physical interaction between NEUROD1 and BRD4 remained intact despite JQ1 treatment, suggesting that BETi blocks NEUROD1 transactivation by inhibiting BET bromodomain proteins instead of disrupting the interaction between these two proteins. Taken together, these results demonstrated that BETi suppresses the NEUROD1 gene network, especially the genes with NEUROD1-loaded SEs.

\section{NEUROD1-subtype of SCLC lines are sensitive to BETi.}

Using the CRISPR screen results from the DepMap project [22, 23], we found that NEUROD1 is a high dependency gene in the NEUROD1-subtype SCLC lines (Fig. 5A). We predicted that inhibition of NEUROD1 transcriptional activity by BETi would significantly affect cell growth, and loss of NEUROD1 would render cells resistant to BETi. To test this, we examined the sensitivity of H446 and DMS-273 NEUROD1-KO cells to JQ1. Compared to control cells (average $\mathrm{IC}_{50} 0.5 \mu \mathrm{M}$ ), the $\mathrm{IC}_{50}$ value to JQ1 was more than 20-fold higher in H446 NEUROD1KO cells (average $\mathrm{IC}_{50}>10 \mu \mathrm{M}$; Fig. 5B). We observed a similar increase in the $\mathrm{IC}_{50}$ value to JQ1 in the DMS-273 NEUROD1-KO cells $(2.5 \mu \mathrm{M})$ compared to the corresponding control and parental cells (both were $0.15 \mu \mathrm{M}$; Fig. 5C). To examine whether turning on NEUROD1 in nonNEUROD1 subtypes of SCLC lines would increase their sensitivity to BETi, we stably overexpressed NEUROD1 in H1048 (a POU2F3-subtype SCLC line) and DMS-114 (a YAP1subtype SCLC line). Despite successful overexpression of NEUROD1, we could not activate NEUROD1-target genes or change the sensitivity of these cells to BETi, likely because of high 
expression levels of REST (a transcriptional repressor of neural genes [24]) in both cell lines (data not shown).

A

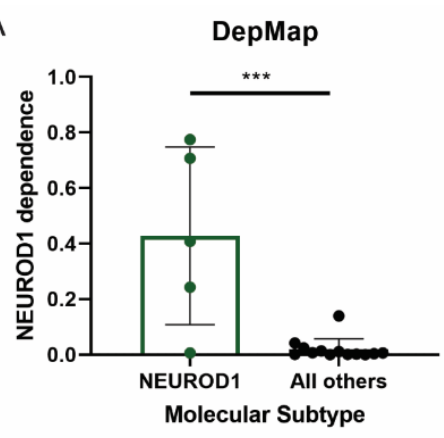

D

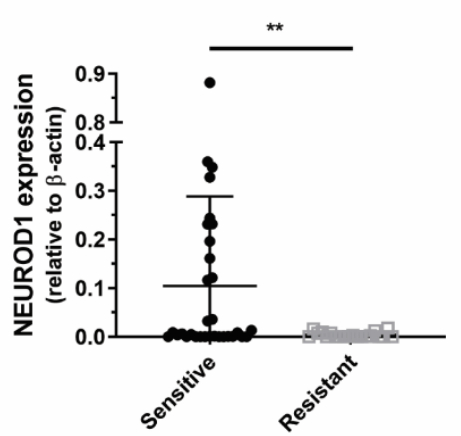

B

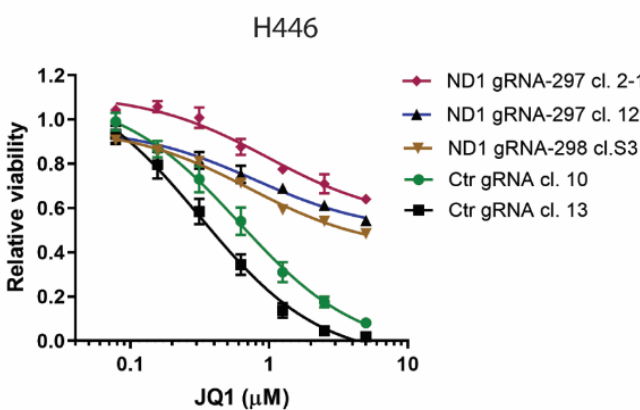

C

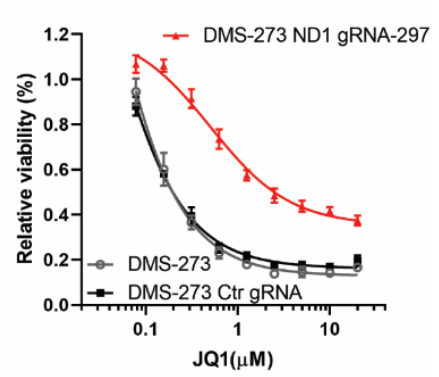

E

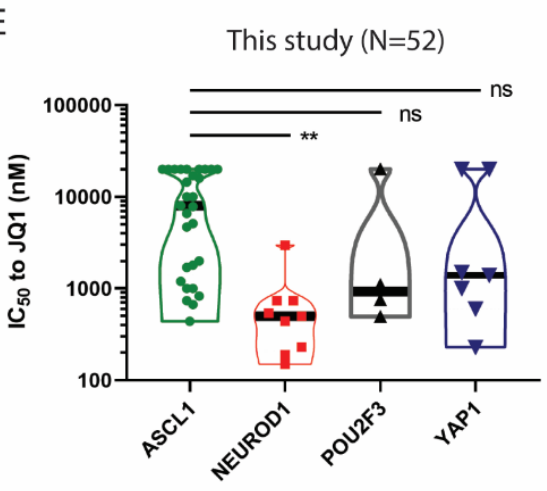

SCLC subtypes
$\mathrm{F}$

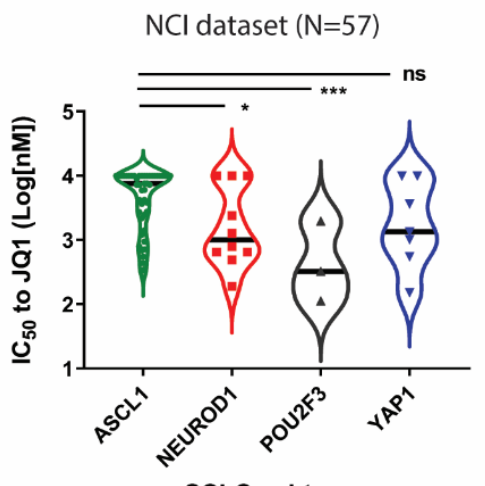

Figure 5. NEUROD1-subtype SCLC lines are sensitive to BETi. A) Comparison of NEUROD1 dependence between the NEUROD1-subtype lines versus all other SCLC lines, using the Achilles Gene Dependency results from the DepMap project (2020 Q2). B) Viability of the H446 NEUROD1-KO cells (gRNA-297 cl. 12 and cl. 2-1; gRNA-298 cl. S3) versus control cells (Ctr gRNA cl. 10 and cl. 13) after JQ1 treatment (72 hrs). C) Viability of the DMS-273 NEUROD1KO cells versus control and parental cells after JQ1 treatment (72 hrs). D) Comparison of relative NEUROD1 expression between the JQ1-sensitive and -resistant SCLC lines $(\mathrm{N}=52)$. An $\mathrm{IC}_{50}$ to $\mathrm{JQ} 1=5 \mu \mathrm{M}$ was used as a cutoff to define the sensitive and resistant SCLC lines. E) Comparison of JQ1 sensitivity $\left(\mathrm{IC}_{50}\right)$ among four molecular subtypes of SCLC lines used in this study $(\mathrm{N}=52)$. F) Comparison of JQ1 IC 50 values among four molecular subtypes of SCLC lines $(\mathrm{N}=57)$ in an NCI drug screen dataset [25]. The significance of the two-group comparisons was determined using the Student's $t$-test in (A) and (D) and the ANOVA test with Dunnett's multiple test correction in $(\mathrm{E})$ and $(\mathrm{F}) .^{*}, \mathrm{P}<0.05 ; * *, \mathrm{P}<0.01$; ***, $\mathrm{P}<0.001$; ns: not significant. The error bars 
in (B-C) represent SD; the top and bottom whiskers in (A) and (D) represent the first and third quartile, respectively; and the thick black lines in $(\mathrm{E})$ and $(\mathrm{F})$ represent the medians.

To examine whether NEUROD1 expression predicts BETi sensitivity, we measured the $\mathrm{IC}_{50}$ values to JQ1 and NEUROD1 expression levels in 52 SCLC lines (Table S14). We divided SCLC lines into two groups based on their sensitivity to JQ1, using $\mathrm{IC}_{50}$ to JQ1 $=5 \mu \mathrm{M}$ as the cutoff. NEUROD1 was expressed only in the cell lines sensitive to JQ1 (Fig. 5D). Based on the expression of ASCL1, NEUROD1, POU2F3, and YAP1, we divided all 52 SCLC lines into four molecular subtype groups (Table S1). In general, the SCLC lines in the NEUROD1-subtype group were more sensitive to BETi than those in the ASCL1-subtype group (Fig. 5E). The SCLC lines in the POU2F3 and YAP1 groups were too few for meaningful comparison. We used an independent dataset (NCI drug screen dataset [25]) and confirmed that the NEUROD1-subtype SCLC lines were more sensitive to JQ1 than the ASCL1-subtype lines (Fig. 5F). Collectively, these results demonstrate that the NEUROD1-subtype SCLC lines are susceptible to BETi.

\section{LSAMP, a NEUROD1-target gene, mediates sensitivity to BETi.}

To identify the genes that mediate the sensitivity of NEUROD1-subtype SCLC lines to BETi, we focused on the NEUROD1-target genes that had $>2$-fold change in expression after BETi treatment in H446 cells. We manually selected 45 genes that showed a substantial loss of BET bromodomain proteins and NEUROD1 occupancy at their gene loci upon the KO of NEUROD1 for a siRNA screen (Fig. 6A; Table S15). We performed the screen in H446 and CORL279 cells, and 13 and 14 genes consistently had $\geq 20 \%$ growth inhibition after a knockdown by two different siRNAs, respectively (Fig. 6B; Table S16). Among the ten genes in common between the two cell lines (highlighted in red), we decided to focus on LSAMP (limbic system-associated 
membrane protein), whose expression was associated with a worse prognosis in SCLC patients (Fig. 6C).

Our ChIP-seq and RNA-seq results indicate that both NEUROD1 and BET bromodomain proteins regulate $L S A M P$ (Fig. S7A-B). We performed western blots to confirm these results. As shown in Figure 6D, LSAMP could only be detected in the plasma membrane fraction but not in the cytosolic fraction, consistent with its role as a neuronal surface glycoprotein. Furthermore, LSAMP expression was lost in the H446 and DMS-273 NEUROD1-KO cells, compared to their corresponding control or parental cells (Fig. 6D-E). We found JQ1 decreased LSAMP expression in a dose-dependent manner in H446 cells (Fig. 6F). These results confirmed that LSAMP is regulated by both NEUROD1 and BET bromodomain proteins.

To validate the findings of the siRNA screens that LSAMP is important for cell survival, we used two siRNAs different from the ones used in the screens to knock down LSAMP (Fig. 6G). We confirmed growth inhibition in H446 cells after knocking down LSAMP (Fig. 6H). To further study the function of LSAMP, we stably overexpressed LSAMP in COR-L279, which has a much lower basal level of this protein than H446 (Fig. 6I). In the initial five days after seeding, the CORL279 cells with ectopic LSAMP grew at the same rate as the cells with a control vector and the parental cells; however, the former cells continued to proliferate beyond day five while the last two cells ceased growing and had lower viability (Fig. 6J). In addition, the COR-L279 cells with ectopic LSAMP were less sensitive to BETi at high doses than control cells (Fig. 6K). We suspected that the forced expression of LSAMP made the cells less susceptible to apoptosis. In support of this notion, the COR-L279 cells with ectopic LSAMP had less cleavage of caspase 3 and 9 than control cells after JQ1 treatment (Fig. 6L). Taken together, these results suggest that suppression of NEUROD1-target genes contributes to BETi sensitivity. 
A

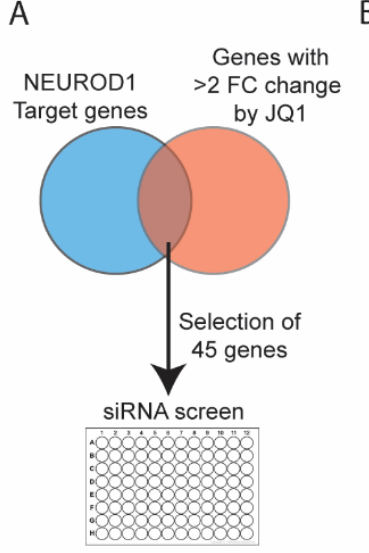

B

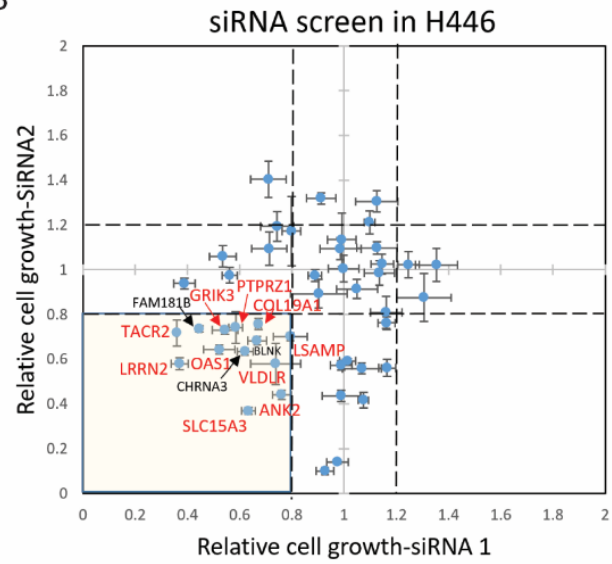

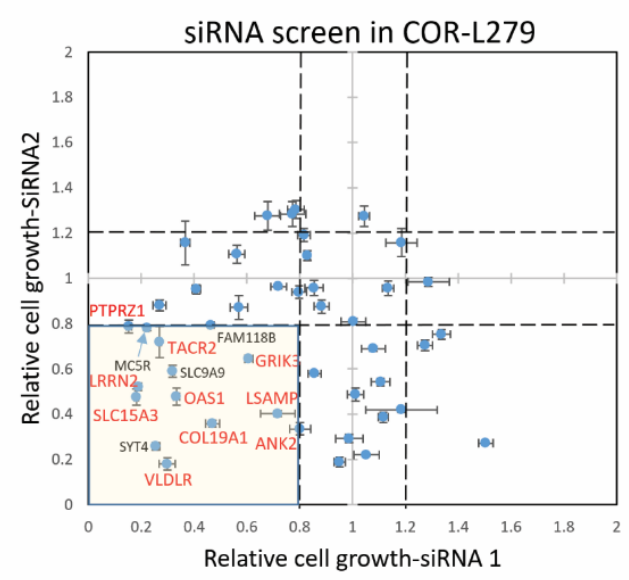

$\mathrm{E}$
C

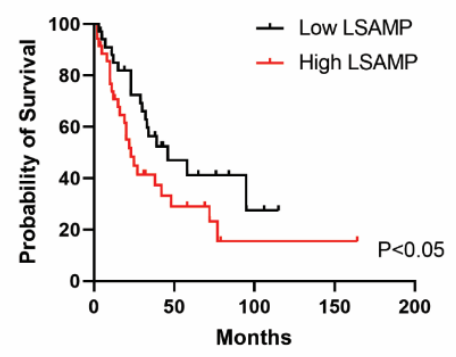

$\mathrm{F}$

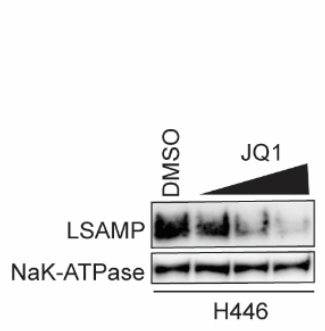

J

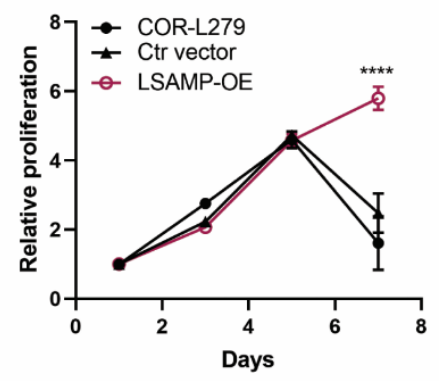

D

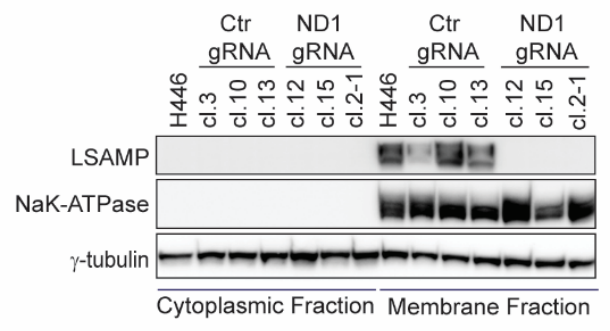

$\mathrm{H}$
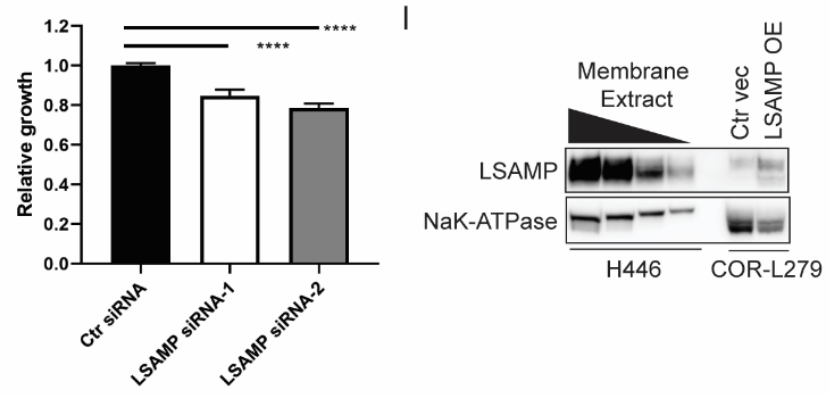

$\mathrm{L}$

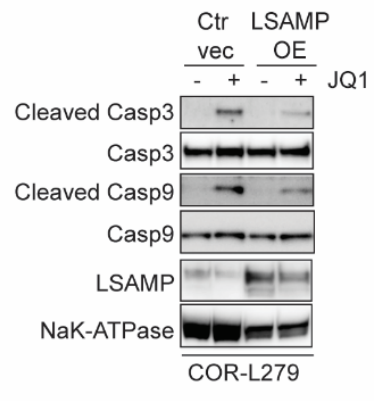

Figure 6. $L S A M P$, a NEUROD1-target gene, mediates sensitivity to BETi. A) A diagram showing the selection of 45 genes for a custom siRNA screen. B) Results of the siRNA screen in H446 (left) and COR-L279 cells (right). The genes highlighted are the common genes in H446 and COR-L279 cells that showed consistently $\geq 20 \%$ growth inhibition upon knockdown by two different siRNAs. C) An inverse correlation in the Kaplan-Meier curve between the LSAMP 
expression and the overall survival of the SCLC patients [26]. The significance of overall survival difference when comparing the patients with top 50\% LSAMP expression versus those with the bottom 50\% was determined using the Log-rank test. D-E) Drastic decrease of LSAMP expression in the NEUROD1-KO cells of H446 (D) and DMS-273 (E). NaK-ATPase serves as a loading control for membrane proteins. F) A dose-dependent decrease of membrane LSAMP expression in H446 cells treated with JQ1 (24 hrs at $0.25,0.5$, or $1 \mu \mathrm{M})$. G) Knockdown of LSAMP in H446 cells using two siRNAs different from the ones in the initial screen (B). H) Significant decrease in cell growth after knockdown of LSAMP in H446 cells using two siRNAs. I) Stable ectopic expression of LSAMP in COR-L279 cells. For comparison, a tapering amount of H446 membrane protein $(20,10,5,2.5 \mu \mathrm{g}$ protein) and $20 \mu \mathrm{g}$ COR-L279 membrane protein were loaded. J) Relative growth of the COR-L279 cells with ectopic LSAMP versus cells transfected with an empty vector or parental cells. K) Viability of the COR-L279 cells with ectopic LSAMP versus control cells after JQ1 treatment (72 hrs). L) Comparison of caspase 3 and 9 cleavages after JQ1 treatment $(5 \mu \mathrm{M}, 24 \mathrm{hrs})$ in the COR-L279 cells with ectopic LSAMP versus control cells. The significance of the two-group comparisons was determined using the Student's $t$-test in $(\mathrm{K})$ and the ANOVA test with Dunnett's multiple test correction in $(\mathrm{H})$ and $(\mathrm{J})$. Where indicated, **, $\mathrm{P}<0.01$; ****, $\mathrm{P}<0.0001$. The error bars represent SD. Ctr, control; FC, fold change; gRNA, guide RNA; OE, overexpression; siRNA, small interfering RNA.

\section{BETi inhibits SCLC growth by suppressing the NEUROD1 gene network in vivo.}

To examine the therapeutic potential of BETi in vivo, we chose to use NHWD-870, a potent BETi in early phase clinical studies, for its favorable in vivo pharmacokinetics compared to JQ1 [27]. We first confirmed that NHWD-870 has similar sensitivity profiles as JQ1 in SCLC lines

(Fig. S8). We evaluated the anti-tumor effects of NHWD-870 in a NEUROD1-subtype SCLC patient-derived xenograft (PDX) model, LX22. As shown in Figure 7A-B, NHWD-870 significantly decreased tumor burden and extended median survival of the tumor-bearing mice from 27 days to 42 days. To assess how NHWD-870 affected NEUROD1-target genes in LX22 tumors, we used RNA-seq to profile the tumor cells isolated from the xenografts after NHWD-870 
treatment (daily for five days; Table S17). As shown in Figure 7C, NHWD-870 significantly depleted the NEUROD1 gene signature in the xenografts compared to vehicle. We confirmed that NHWD-870 decreased the LSAMP transcripts but did not affect the NEUROD1 expression (Fig. 7D).

A

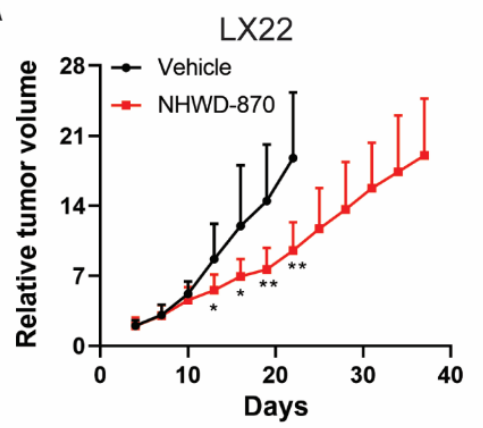

D

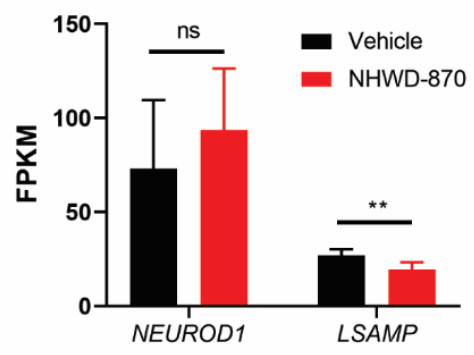

G

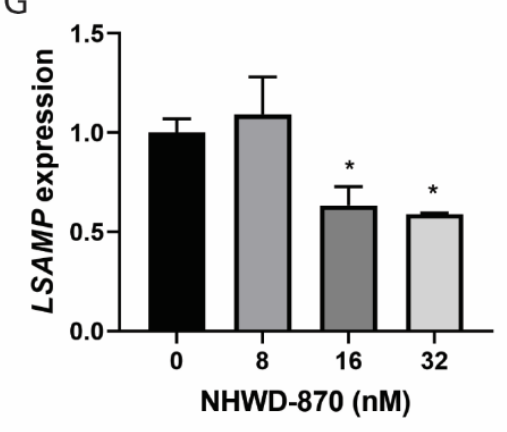

B

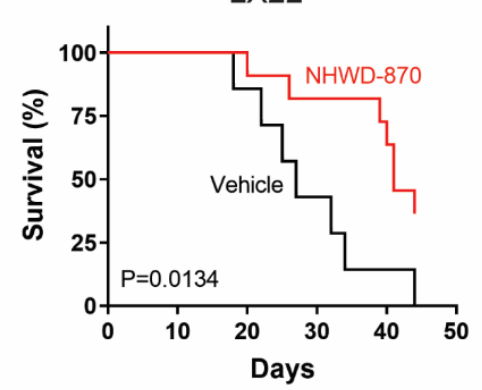

$E$

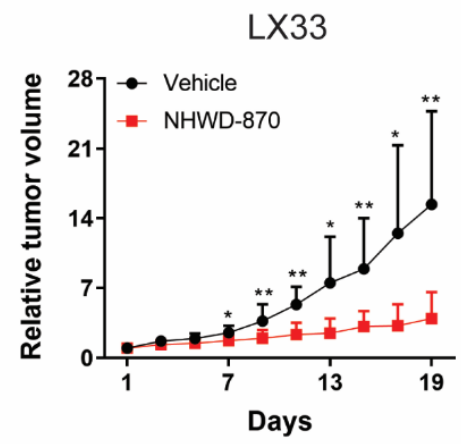

$\mathrm{H}$

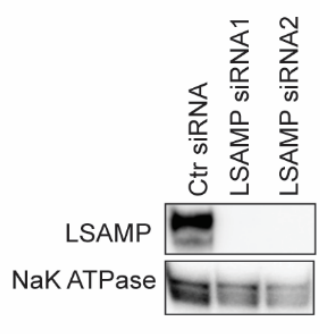

C

LX22

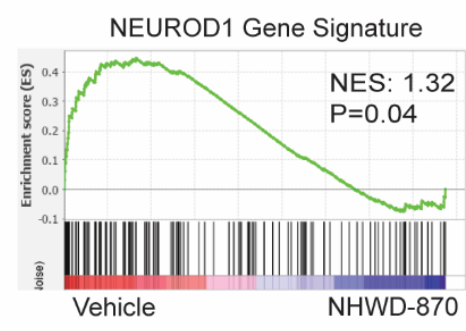

$\mathrm{F}$
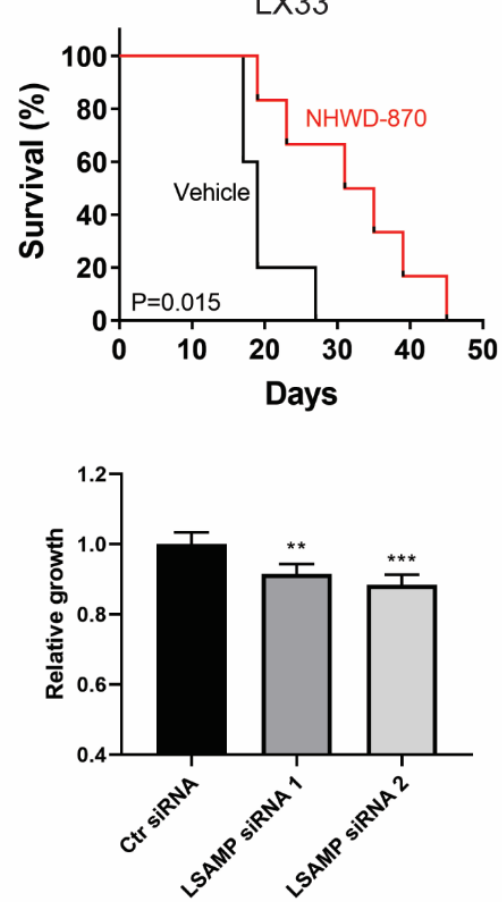

Figure 7. BETi inhibits SCLC growth by suppressing the NEUROD1 gene network in vivo. A) NHWD-870 (3mg/kg; oral gavage on a 5-day-on/2-day-off schedule) suppressed tumor growth in a NEUROD1-subtype SCLC PDX model - LX22. n=13 per group. B) The Kaplan-Meier survival curve of (A). C) GSEA plot of the NEUROD1 gene signature comparing the LX22 PDX tumors treated with NHWD-870 (3mg/kg; daily for five days) to control treated with the vehicle. $\mathrm{n}=6$ per group. D) Comparison of NEUROD1 and LSAMP transcript abundance in LX22 tumors treated 
with NHWD-870 versus vehicle. E) NHWD-870 (3mg/kg; oral gavage daily) suppressed tumor growth in LX33, another NEUROD1-subtype SCLC PDX model. n=6 per group. F) The KaplanMeier survival curve of (E). G) A dose-dependent decrease in LSAMP expression in the LX33 SCLC line after exposure to NHWD-870 for 24 hrs. H) Knockdown of LSAMP in the LX33 SCLC line using two different siRNAs. I) Relative growth of the LX33 SCLC cells after knockdown of LSAMP. The significance of the two-group comparison was determined using the Student's $t$-test in (A), (D), and (E), the Log-rank test in (B) and (F), and the ANOVA test with Dunnett's multiple test correction in $(\mathrm{G})$ and $(\mathrm{I})$. Where indicated, *, $\mathrm{P}<0.05$; **, $\mathrm{P}<0.01$; ***, $\mathrm{P}<0.001$; ns, not significant. The error bars represent SD. Ctr, control; FPKM, Fragments Per Kilobase of transcript per Million mapped reads; NES, normalized enrichment score.

We next tested NHWD-870 in another NEUROD1-subtype SCLC PDX, LX33. Similar to LX22, NHWD-870 significantly lessened the tumor burden and extended the median survival of the mice from 20 days to 32 days in the LX33 model (Fig. 7E-F). For the further mechanistic study, we attempted to establish SCLC cell lines from the LX22 and LX33 PDXs but were only successful with the latter. In the LX33 SCLC line, we found NHWD-870 suppressed LSAMP at a dose as low as $16 \mathrm{nM}$ (Fig. 7G). To examine if LSAMP is essential for the growth of the LX33 SCLC, we knocked down LSAMP using two different siRNA (Fig. 7H). As shown in Figure 7I, the knockdown of LSAMP decreased the growth of the LX33 cells. Taken together, these findings demonstrate that suppression of the NEUROD1 gene network contributes to the BETi-induced growth inhibition of SCLC in vivo.

\section{DISCUSSION}

There is growing evidence that primary SCLC tumors are heterogeneous [28, 29], and it is appealing to develop personalized therapies to target specific molecular subtypes to improve precision $[6,30]$. In this context, we present evidence that the NEUROD1-subtype SCLC is more 
sensitive to BETi. This susceptibility stems from the dependence of NEUROD1 on BET bromodomain proteins to activate its downstream gene network. Our findings suggest that targeting a coactivator could meaningfully block the function of a master transcription factor in SCLC, which reveals a new potential approach to develop subtype-specific therapies.

Our results provide epigenomic evidence supporting that NEUROD1 is a master transcription factor in a subset of SCLC. In H446 cells, the NEUROD1-loaded SEs colocalized with $80 \%$ of the BRD4-loaded SEs, and KO of NEUROD1 caused drastic genome-wide alterations in H3K27Ac and BET bromodomain protein occupancy. With our further findings that NEUROD1 physically interacts with BET bromodomain proteins, we conclude that NEUROD1 defines the landscape of enhancer and SE in the NEUROD1-subtype SCLC.

The susceptibility of NEUROD1-target genes to BETi indicates that NEUROD1 depends on BET bromodomain proteins for its transactivation. In the NEUROD1-KO cells, the landscape of BET bromodomain proteins is drastically different, and these factors now regulate a mostly different set of genes. Although BETi was equally effective in suppressing this new set of genes, the drug resistance in the NEUROD1-KO cells suggests that these genes were not critical for cell survival.

In search of NEURDO1-target genes essential for cell survival, we identified LSAMP as a potential candidate, despite that many other NEUROD1-target genes may also contribute to cell growth as suggested by the siRNA screen results. LSAMP is a member of the IgLON family of immunoglobulin domain-containing cell adhesion molecules. It was speculated as a tumor suppressor gene in ovarian cancer and osteosarcoma, based on its frequent deletion in the tumors and the correlation between its low expression and worse prognosis $[31,32]$. The survival analysis in SCLC patients and the in vitro findings in this study led us to draw an opposite conclusion, 
suggesting that the function of LSAMP could be tumor type-specific. Our results indicate that LSAMP antagonizes apoptosis in SCLC, but the detailed mechanism awaits future investigation.

Our results and those from a public dataset demonstrate that, despite a wide range of sensitivity in the ASCL1-subtype SCLC lines, these cells are generally more resistant to BETi than the NEUROD1-subtype lines. These results contradict a previous report that ASCL1 expression could predict the sensitivity of SCLC to BETi [33]. Although BETi decreases ASCL1 expression as reported previously [33], BETi could inadvertently turn on the insulin-like growth factor 1 (IGF1) pathway in ASCL1-subtype SCLC lines by downregulating IGFBP5, an ASCL1-target gene and a negative regulator of IGF1 signaling [34]. Such a survival mechanism was not present in the NEUROD1-subtype SCLC lines [34], which likely contributes to the differential sensitivity to BETi between these two molecular subtypes of SCLC.

An intriguing finding from our study is that the KO of NEUROD1 triggered SCLC molecular subtype evolution. Recent works from Trudy Oliver's group have demonstrated that knocking in an active form of Myc $\left(M y c^{T 58 A}\right)$ prompted ASCL1-subtype SCLCs to evolve into a NEUROD1- and then a YAP1-subtype [20, 35]. MYC, amplified in H446 and DMS-273 cells (according to the copy number results of the DepMap project), is likely the driving force of subtype evolution in the NEUROD1-KO cells of both cell lines. These findings suggest a model that NEUROD1 counteracts MYC and prevents cells from evolving into a YAP1 subtype, but overactive MYC or knockout of NEUROD1 could tilt this balance and trigger subtype evolution. We observed similar changes in the genes related to NE, EMT, and YAP1 signaling upon NEUROD1 KO in H446 and DMS-273 cells but found differential upregulation of Notch pathway genes. As NOTCH signaling is essential for MYC-driven subtype evolution in SCLC but becomes 
dispensable once the cells had evolved into a YAP1 subtype [20], this signaling was possibly transiently turned on during the subtype evolution of DMS-273 cells upon NEUROD1 KO.

In summary, we demonstrate that BETi targets NEUROD1-subtype SCLC by suppressing the NEUROD1 gene network. Future study is needed to determine whether NEUROD1 could be used as a predictive marker to identify patients with SCLC tumors sensitive to BETi. A deeper understanding of transcription regulation by other master factors in SCLC could yield additional targets to enable precision therapy for this devastating malignancy. 


\section{MATERIALS AND METHODS}

Cell Culture. All SCLC lines used in this study were purchased from the American Type Culture Collection, the German Collection of Microorganisms and Cell Cultures, and the Japanese Collection of Research Bioresources Cell Bank (Table S1). Cells were cultured in media recommended by the vendors and were maintained in a humidified incubator at $37^{\circ} \mathrm{C}$ with $5 \%$ $\mathrm{CO}_{2}$. LX33 SCLC line was established by growing single cells isolated from an LX33 PDX tumor in HITES media. All cell lines tested negative for mycoplasma. Short tandem repeat analysis was performed to authenticate select cell lines.

CRISPR Knockout. CRISPR was performed using a two-step method [36]. Briefly, a Cas9 expression vector, lentiCas9-Blast (Addgene, \#52962), was transfected into H446 and DMS-273 cells through lipofection. After three days, the transfected H446 and DMS-273 cells were selected with 2.5 and $1 \mu \mathrm{g} / \mathrm{ml}$ blasticidin (Gibco) to establish cell clones stably expressing cas9, respectively. One clone with the highest Cas9 expression was chosen in each cell line for viral transduction at a multiplicity of infection of 2 in the presence of $5 \mu \mathrm{g} / \mathrm{ml}$ polybrene (Sigma). The lentiviral particles of gRNA-carrying vectors targeting NEUROD1 (Sigma) were HS5000024297 (abbreviated as \#297, GAG TCC TCC TCT GCG TTC ATG G) and HS5000024298 (abbreviated as \#298, GAG GAG GAG GAC GAA GAT GAG G). Three days after viral transduction, the H446-cas9 and DMS-273-cas9 cells were subject to selection with 2.5 and $0.5 \mu \mathrm{g} / \mathrm{ml}$ puromycin (Gibco), respectively. Individual clones were expanded and verified for KO of NEUROD1. The clones were maintained in media without selection antibiotics. 
Sanger Sequencing. The genomic DNA was extracted from cells using a Quick-DNA kit (Zymo Research). After quantification using a Nanodrop One (Thermo Scientific), 100ng genomic DNA was used to amplify the DNA fragments around the CRISPR targets using a Herculase II fusion DNA polymerase kit (Agilent). The following primers were used: \#297 target, sense 5'-GCC TCT CCC TTG TTG AAT GT-3', antisense 5'-TCA TCC TCC TCT TCC TCT TCT T-3'; \# 298 target, sense 5'-CGC AAG CAT TTG TAC AGG TTT AG-3', antisense 5'- GCG AGC CTT AGT CAT CTT CTT C-3'. The PCR products were purified using a PCR purification kit (Qiagen) and were subject to Sanger sequencing (Genewiz).

RNA Isolation and Quantitative Reverse Transcription-PCR Assay. RNA was extracted from cells using an RNeasy Mini Kit (Qiagen) and quantified using a Nanodrop. Equal amounts of total RNA were used for cDNA synthesis using an iScript cDNA Synthesis Kit (Bio-Rad) or a LiScript First-Strand cDNA Synthesis Kit (LifeSct). Quantitative gene expression analysis was performed on an ABI PRISM7500 real-time PCR system (ThermoFisher), using a SsoAdvanced ${ }^{\text {TM }}$ Universal SYBR $^{\circledR}$ Green Supermix kit (Biorad) and specific primers (Biorad or Integrated DNA Technologies).

RNA Sequencing and Data Analysis. Total RNA libraries (COR-L279) and mRNA libraries (H446 and LX22) were constructed using a TruSeq Stranded Total RNA Kit (Illumina, RS-1222201) and an mRNA Library Prep kit V2 (Illumina, RS-122-2001), respectively. Paired-end sequencing of the constructed libraries was performed on a HiSeq 4000 system (Illumina).

The fastq files from the high-throughput sequencing were uploaded to the NIH's Biowulf cluster and analyzed using the CCBR/Pipeliner [37]. Briefly, the sequencing quality was assessed 
using FastQC, and adapter sequences and low-quality base pairs were trimmed using Cutadapt. Trimmed reads were then mapped to the human hg19 reference genome using STAR, and gene expression was quantified using RSEM. Differential gene expression analysis was performed using limma. A false-discovery rate of 0.05 was set as a cutoff to define significant alterations in gene expression.

Gene Set Enrichment Analysis (GSEA). GSEA was performed using GSEA (v.4.0.2) under the setting of gene_set permutation at 1,000 times. DESeq2 normalized counts were used as data input. Normalized enrichment score (NES) and FWER P-value were shown for each GSEA plot. Two functional gene sets from the Molecular Signature Database were used: "Cordenonsi YAP1 Conserved Signature" (M2871) and "Hallmark Epithelial-Mesenchymal Transition Signature" (M5930). The "Neuroendocrine gene signature" included the 25 genes previously reported to be positively associated with neuroendocrine differentiation in SCLC cell lines [38]. The "NEUROD1 gene signature" was created using 384 NEUROD1-target genes showing at least 4fold gene expression changes (H446 NEUROD1-KO cells versus control cells; Table S7).

ChIP-seq. ChIP-seq was performed as described previously [39]. Briefly, H446 NEUROD1-KO cells (cl. 2-1) and control cells (cl. 10) were crosslinked with 1\% formaldehyde (Sigma) for $10 \mathrm{~min}$ and quenched by $125 \mathrm{mM}$ glycine (Sigma) for $5 \mathrm{~min}$. After washing cells with ice-cold PBS twice, Farnham lysis buffer (5 mM PIPES, pH 8.0, $85 \mathrm{mM} \mathrm{KCl,} \mathrm{and} \mathrm{0.5 \%} \mathrm{NP-40)} \mathrm{supplemented} \mathrm{with} \mathrm{a}$ protease inhibitor cocktail (Sigma) was added, and the cells were scraped off and pelleted at 2,000 $\mathrm{rpm}$ at $4{ }^{\circ} \mathrm{C}$ for $5 \mathrm{~min}$. The cell pellets were resuspended in Farnham lysis buffer at a concentration of $2 \times 10^{7}$ cells per $\mathrm{ml}$, and the cell suspension was carefully passed through a 20 gauge needle 20 
times without generating air bubbles. The lysates were centrifuged at $2,000 \mathrm{rpm}$ at $4{ }^{\circ} \mathrm{C}$ for $5 \mathrm{~min}$, and the pellets containing nuclei were resuspended with $1 \mathrm{~mL}$ buffer containing $50 \mathrm{mM}$ Tris-HCl, pH 8.0, $10 \mathrm{mM}$ EDTA, 0.1\% SDS, and protease inhibitor cocktail. After sonication, the solution was centrifuged at $13,000 \mathrm{~g}$ at $4{ }^{\circ} \mathrm{C}$ for $10 \mathrm{~min}$. The supernatant containing sheared chromatin was supplemented with $1 \%$ Triton-X100, 0.1\% sodium deoxycholate, and a protease inhibitor cocktail. Two percent of the mixture was set aside as input. For each ChIP, $4-10 \mu \mathrm{g}$ of antibodies were added ( $\alpha$-NEUROD1, Cell Signaling Technology, \#4373; $\alpha-H 3$ K27Ac, Abcam, \#ab4729; $\alpha$-BRD2, Cell Signaling Technology, \#5848; $\alpha$-BRD3, Bethyl, A302-368A; $\alpha$-BRD4, Bethyl, A700-004; $\alpha$ RPII, Millipore, \# 05-952-I). After mixing on a rotator at $4{ }^{\circ} \mathrm{C}$ overnight, $50 \mu \mathrm{L}$ prewashed protein A or G Dynabeads (ThermoFisher) were added, followed by mixing on a rotator for additional $3 \mathrm{hrs}$ at $4^{\circ} \mathrm{C}$. Bead-antibody-chromatin complexes were collected on a magnetic rack and washed with $1 \mathrm{ml}$ cold RIPA buffer $\mathrm{x} 2,1 \mathrm{ml}$ cold RIPA buffer containing $300 \mathrm{mM} \mathrm{NaCl}$ x2, $1 \mathrm{ml}$ cold LiCl buffer x2, and PBS x1. Antibody-chromatin complexes were eluted from the beads with $100 \mu \mathrm{L}$ buffer containing $0.1 \mathrm{M} \mathrm{NaHCO} 3$ and 1\% SDS, and the elution was supplemented with $20 \mu \mathrm{g}$ Proteinase $\mathrm{K}$ and incubated at $65^{\circ} \mathrm{C}$ overnight. Input sample volumes were adjusted to $100 \mu \mathrm{L}$ with the elution buffer and processed with the ChIPed samples. The DNA was then purified using QIAquick PCR purification kit (Qiagen) and eluted in $30 \mu \mathrm{L} 10 \mathrm{mM}$ Tris-HCl. Libraries were constructed using entire ChIPed DNA or 300 ng of the input DNA using a NEBNext Ultra II DNA Library Prep kit with AMPure XP magnetic beads (Beckman Coulter). Estimation of library quality and quantity was performed using a Bioanalyzer and Qubit assays, and the libraries were sequenced using a HiSeq 3000 system (Illumina). 
ChIP-seq - Peak Calling. Initial quality check of the fastq files was performed using FastQC, and adapter sequences and low-quality base pairs were trimmed using Cutadapt. Trimmed reads were mapped to the human hg19 reference genome using the backtrack algorithm of Burrows-Wheeler Aligner (BWA-Backtrack) [40]. Duplicated reads were filtered, and the peaks were called using Model-based Analysis of ChIP-Seq (MACS) with the corresponding input as control [41]. Peaks of RPII, BRD2, BRD3, and BRD4 were called using default parameters, and H3K27Ac and NEUROD1 peak calling were performed using the parameters optimized for histone and transcription factor, respectively [41]. Genomic annotations of the NEUROD1 peaks were performed using the 'annotatePeaks' function of the Homer v4.11 [42].

ChIP-seq - Metagene Analysis. The metagene profile of the NEUROD1 peaks was performed using the 'makeMetaGeneProfile' function of the Homer. The NEUROD1 peaks identified by MACS were used as the input.

ChIP-seq - Peak Colocalization. Tag directories were first generated from the bam file of each ChIP-seq sample and the corresponding input, using the 'makeTagDirectory' function of the Homer. Histograms on the occupancy of H3K27Ac and BET bromodomain proteins within 2.5KB of the NEUROD1 peaks were produced using the 'annotatePeaks' function of the Homer with the parameter '-size 5000 -hist 10'. The results were plotted using GraphPad Prism (v8.4.3).

The genomic positions of TEAD1, TEAD4, ZEB1, and RBPJ peaks were downloaded from ChIP-atlas.org [43]. TEAD1 genomic positions were extracted from the pooled TEAD1 peaks in MSTO-211H cells (a mesothelioma line; GSM1664955; [44]) and H69 cells (a human intrahepatic duct line; GSM1524339; [44]); TEAD4 genomic positions were the pooled peaks from A549 cells 
(a non-small cell lung cancer line; GSM1010868; [45]) and PC9 cells (a non-small cell lung cancer line; GSM3813968, GSM3813971, GSM3813975, GSM3813978; [46]); ZEB1 genomic positions were from H1975 cells (a non-small cell lung cancer cell line; GSM2857242 and GSM2857243; [47]) and RBPJ genomic positions were from GSC8 cells (a glioblastoma cell line; GSM1922946, GSM1922947, and GSM1922948; [48]).

ChIP-seq - Heatmap. BRD4 peaks colocalizing with NEUROD1 peaks were identified using Homer's 'mergePeaks' function with the setting '-d given'. Peak annotation was performed using the 'annotatePeaks' function of Homer. The NEUROD1 or BRD4 occupancy near the BRD4 peaks overlayed with or separated from the NEUROD1 peaks were assessed using Homer's 'annotatePeaks' function. The results were visualized in heatmaps using EaSeq [49].

ChIP-seq - de novo Motif Discovery. Motif discovery was performed using the 'findMotifsGenome' function of Homer v4.11, with the setting of '-size 200 -mask'. The NEUROD1 peaks identified by MACS were used as the input.

ChIP-seq - Superenhancer (SE) Analysis. The H3K27Ac peaks were used to mark constituent enhancer sites in the H446 control cells and the NEUROD1-KO cells, and BRD4-loaded or NEUROD1-loaded SEs were called by ROSE using the default setting [19, 50]. The identified SEs were annotated for the nearest genes using the 'annotatePeaks' function of Homer.

Identification of NEUROD1-Target Genes. The NEUROD1-target genes were identified using the Binding and Expression Target Analysis (BETA) algorithm [21]. The input data were the 
NEUROD1 peaks identified by MACS and the differential gene expression output from Limma comparing the H446 NEUROD1-KO cells and control cells. The 'Plus' option was used with the setting of '--df 0.05 --bl --da 0.5 ', which limits the analysis to the top 50 percent of differentially expressed genes with FDR $<0.05$ and applies CTCF boundary to limit the number of genes in association with a peak. The output files included a prediction of the NEUROD1 function in transcription and a list of NEUROD1-target genes.

Western Blot Analysis. Suspension cells were collected by centrifugation and washed with PBS once. Adherent cells were washed with PBS right after removing growth media. Cells were lysed in ice-cold 1X RIPA lysis buffer (Millipore) supplemented with a protease inhibitor cocktail. After incubation on ice for $10 \mathrm{~min}$, the lysed cells were centrifuged at $10,000 \mathrm{~g}$ for 10 minutes to collect cell lysates. Membrane proteins were extracted from cells using a Mem-PER Plus Membrane Protein Kit (ThermoFisher).

Protein quantification was performed using a Pierce BCA Protein Assay Kit (ThermoFisher). Equal amounts of protein lysates were resolved on precast polyacrylamide denaturing gels (ThermoFisher or GenScript). Proteins were transferred onto 0.2 $\mu \mathrm{m}$ PVDF membranes using either a dry transfer with the iBLOT2 system (ThermoFisher) or a conventional wet transfer. After blocking the membranes with TBST $+5 \%$ non-fat dried milk, membranes were incubated with primary antibodies, NEUROD1 (BD Biosciences, \#563000, 1:2,000), YAP1 (Santa Cruz Biotechnology, \#sc-101199, 1:200), BRD2 (Santa Cruz Biotechnology, \#sc-393720, 1:200), BRD3 (Santa Cruz Biotechnology, \#sc-81202, 1:200), BRD4 (Novus, NBP2-52959, 1:1,000), LSAMP (Abcam, \#ab89719, 1:1,000), NaK-ATPase (Santa Cruz Biotechnology, \#sc-71638, 1:1,000), cleaved caspase 3 (Cell Signaling Technology, \#9661, 
1:1,000), caspase 3 (Cell Signaling Technology, \#9662, 1:1,000), cleaved caspase 9 (Cell Signaling Technology, \#7237, 1:1,000), caspase 9 (Cell Signaling Technology, \#9502, 1:1,000), $\gamma$-tubulin (Sigma, \#T6557, 1:10,000). After incubating the membranes with an appropriate secondary antibody, the presence of a protein of interest was detected by chemical fluorescence following a conventional ECL Western blotting protocol.

siRNA Transfection and siRNA Library Screen. siRNAs targeting YAP1 (\#S536627 and \#536628) and LSAMP (\#S8299 and \#S8301) were purchased from Thermo Scientific. The siRNA library that contained 90 siRNA targeting 45 genes of interest (Table S15) was custom synthesized at Qiagen. After resuspending siRNA with RNase-free water, cell transfection was performed using Lipofectamine RNAiMax (Thermo Scientific) with a final working concentration of 20nM siRNA. Three days after transfection, cells were lyzed for protein extraction or cell viability test.

Plasmid Transfection and Establishment of Stable Transformant. Plasmids were transfected into cells using Lipofectamine 3000 reagent (Thermo Scientific) following a standard protocol. Blasticidin was added three days after transfection to select cas9-stable transformants. For the establishment of LSAMP stable transformants, COR-L279 cells were trypsinized three days after transfection. After eliminating large cell clumps by passing cell suspensions through sterile $40 \mu \mathrm{m}$ nylon mesh cell strainers (Fisher Scientific, Hampton, NH), $0.5 \mathrm{ml}$ cell suspension containing 20,000/ml, 2,000/ml, or 200/ml cells was mixed with 2.4 ml ClonaCell ${ }^{\text {TM}}$-TCS medium (Stem Cell Technology), $0.3 \mathrm{ml}$ penicillin/streptomycin, and $60 \mu 1 \mathrm{G} 418$ solution (final concentration: $1 \mathrm{mg} / \mathrm{ml}$; Roche). The mixtures were transferred to a 6-well plate through 16-gauge needles. After threeweek incubation, the surviving cell clones were picked from the semi-solid media using pipet tips. 
The cell clones were expanded in RPMI complete media supplemented with $1 \mathrm{mg} / \mathrm{ml} \mathrm{G} 418$. After verification of LSAMP overexpression, the cells were maintained in media with $0.4 \mathrm{mg} / \mathrm{ml} \mathrm{G} 418$.

Co-immunoprecipitation (Co-IP) Assays. Nuclear extracts were prepared from H446 and H524 cells using a Nuclear Complex Co-IP Kit (Active Motif, \#54001). Each IP, in a total volume of $500 \mu 1$, contained $200-500 \mu \mathrm{g}$ nuclear extract, a specific antibody against the protein of interest ( $\alpha$ BRD4, Bethyl, A301-985A, $5 \mu \mathrm{g} ;$ aNEUROD1, Cell Signaling Technology, \#4373, $10 \mu 1$; $\alpha B R D 2$, Cell Signaling Technology, \#5848, $10 \mu 1)$ and control IgG (Cell Signaling Technology, \#3900, $5 \mu \mathrm{g}$ ), $20 \mu \mathrm{l}$ slurry of Magna ChIP protein A magnetic beads (Millipore), and 1x IP High buffer supplemented with a protease inhibitor cocktail. After overnight mixing on an orbital shaker at $4{ }^{\circ} \mathrm{C}$, antibody/antigen/bead complexes were washed three times with $1 \mathrm{x}$ High Buffer supplemented with $1 \mathrm{mg} / \mathrm{ml}$ bovine serum albumin (BSA), followed by three washes with 1x High Buffer without BSA. The immunoprecipitated protein complexes were eluted by mixing the washed beads with Laemmli buffer supplemented with 5\% 2-mercaptoethanol (Sigma) followed by heating at $95{ }^{\circ} \mathrm{C}$ for $5 \mathrm{~min}$. The presence of the protein of interest was detected by western blot.

Cell Proliferation Assay and Viability Assay. After trypsinization, cell suspensions were passed through $40 \mu \mathrm{m}$ cell strainers, 5000 cells in $100 \mu \mathrm{l}$ growth media were seeded into each well of a 96-well white plate with a clear bottom (Corning, \#3610). Alternatively, 750 cells in $15 \mu 1$ were seeded into each well of a 384-well white plate with a clear base (Corning, \#3765).

For cell proliferation assay, cell viability was checked the next day (D1) and then every two days using CellTiter-Glo ${ }^{\circledR} 2.0$ Reagent (Promega). A reflective foil seal (Bio-Rad, \#MSF1001) 
was applied to the bottom of each plate to maximize the signal. The results were normalized to the reading on D1. Four to five replicates were used for each data point.

For cell viability assay, JQ1 (Sigma) or NHWD-870 (a kind gift from Nenghui Wang) were serially diluted 2-fold in DMSO to generate nine consecutive concentrations. The compounds were first diluted 100 times in culture media, and the diluted drug solution was then delivered to the cells in $10 \%$ of the final volume to achieve a total of 1,000-time dilution. After brief mixing, cells were incubated for 72 hours before cell viability was measured. Four replicates were used for each dose of drug treatment.

Animal Studies. The animal experiments were approved and performed according to the regulations set by the National Cancer Institute-Bethesda Animal Care and Use Committee. SCLC PDX LX22 and LX33 mouse models were kind gifts from Drs. Charles M. Rudin and John T. Poirier [51, 52]. Freshly isolated PDX tumors were dissociated into single-cell suspension using a Human Tumor Dissociation Kit (Miltenyi Biotec) and a gentleMACS ${ }^{\text {TM }}$ Octo Dissociator (Miltenyi Biotec). Afterward, cell suspensions were passed through sterile $70 \mu \mathrm{m}$ cell strainers (Miltenyi Biotec) to remove large cell clumps. Cells were washed with ample amounts of phosphate-buffered saline (PBS) pH 7.2 twice, and red blood cells were lysed by resuspending cells in $5 \mathrm{ml} \mathrm{ACK}$ lysis buffer (Quality Biological) followed by incubation at room temperature for 5 min. Cells were subsequently washed with ample amounts of PBS three times, and an equal amount of cells (about $5 \times 10^{6}$ viable cells) in $100 \mu$ PBS were injected subcutaneously into right flanks of 6-week-old NOD-SCID mice (Charles River Laboratories).

Once the tumor volume reached between 50 and $100 \mathrm{~mm}^{3}$ (average, 14-21 days), mice were randomized, and NHWD-870 or vehicle was given as specified in the figure legends. Animals 
were euthanized if 1) tumor volume was $\geq 1500 \mathrm{~mm}^{3}$; 2) tumor became ulcerated; 3) 75 days had elapsed after a tumor became palpable.

For tumorigenicity assay, H446 NEUROD1-KO cells (cl.12 and cl.15) and control cells (cl.10) were resuspended in Matrigel (Corning) to a final concentration of $1 \times 10^{7}$ cells $/ \mathrm{ml} .100 \mu 1$ cell suspension was injected subcutaneously into each flank of a NOD-SCID mouse. Tumors were resected three weeks (control cells and NEUROD1 gRNA-297 cl.15) or eight weeks after injection (NEUROD1 gRNA-297 cl.12).

RNA Extraction from Tumor Tissues. A magnetic-activated cell sorting method was used to separate tumor cells from mouse stromal cells [53]. In brief, dissociated tumor cells were resuspended in PBS supplemented with $0.5 \%$ bovine serum albumin (Sigma Aldrich) to a final concentration of $2 \times 10^{6}$ cells per $80 \mu$ l. Then, the cell suspension was mixed with $20 \mu 1$ of the cocktail from a Mouse Cell Depletion Kit (Miltenyi Biotec). After 15 min incubation at $4{ }^{\circ} \mathrm{C}$, the mixture was loaded onto an LS column on a MACS Separator (Miltenyi Biotec). The flow-through fraction, which contained enriched human tumor cells, was collected by centrifugation. The cell pellet was processed for RNA isolation using an RNeasy Mini Kit (Qiagen).

Statistical Analysis. Statistical analysis and graphing were performed using GraphPad Prism. Pearson correlation test was used to evaluate the correlation between the $\mathrm{IC}_{50}$ values of JQ1 and NHWD-870. Log-rank (Mantel-Cox) test was employed for Kaplan-Meier survival analysis. The Student's $t$-test was used to compare two groups, and the ANOVA test with Dunnett's multiple comparison correction was applied for multi-group comparison. All tests were performed with a two-sided significance level of 5\%. 
bioRxiv preprint doi: https://doi.org/10.1101/2021.10.25.465771; this version posted October 25, 2021. The copyright holder for this preprint (which was not certified by peer review) is the author/funder. This article is a US Government work. It is not subject to copyright under 17 USC 105 and is also made available for use under a CCO license.

Data availability. All data sets described in the paper are deposited in NCBI Gene Expression Omnibus, and the accession number is pending. 


\section{REFERENCES}

1. National Cancer Institute. Scientific Framework for Small Cell Lung Cancer (SCLC). URL https:/deainfo.nci.nih.gov/advisory/ctac/workgroup/SCLC/SCLC\%20Congressional\%20 Response.pdf. Last visited: 03-12-2021.

2. NCCN Clinical Practice Guidelines in Oncology (NCCN Guidelines): Small Cell Lung Cancer.

$$
\text { Version }
$$$$
3.2021 \text {. }
$$

URL https://www.nccn.org/professionals/physician_gls/pdf/sclc.pdf. Last visited: 03-12-2021.

3. Howlader, N., et al., The Effect of Advances in Lung-Cancer Treatment on Population Mortality. New England Journal of Medicine, 2020. 383(7): p. 640-649.

4. Rudin, C.M., et al., Molecular subtypes of small cell lung cancer: a synthesis of human and mouse model data. Nat Rev Cancer, 2019. 19(5): p. 289-297.

5. Poirier, J.T., et al., New Approaches to SCLC Therapy: From the Laboratory to the Clinic. J Thorac Oncol, 2020. 15(4): p. 520-540.

6. Gay, C.M., et al., Patterns of transcription factor programs and immune pathway activation define four major subtypes of SCLC with distinct therapeutic vulnerabilities. Cancer Cell, 2021.

7. Pang, Z.P., et al., Induction of human neuronal cells by defined transcription factors. Nature, 2011. 476(7359): p. 220-223.

8. Matsuda, T., et al., Pioneer Factor NeuroD1 Rearranges Transcriptional and Epigenetic Profiles to Execute Microglia-Neuron Conversion. Neuron, 2019. 101(3): p. 472-485.e7.

9. Pataskar, A., et al., NeuroD1 reprograms chromatin and transcription factor landscapes to induce the neuronal program. The EMBO Journal, 2016. 35(1): p. 24-45. 
10. Osborne, J.K., et al., NeuroD1 regulates survival and migration of neuroendocrine lung carcinomas via signaling molecules TrkB and NCAM. Proceedings of the National Academy of Sciences, 2013. 110(16): p. 6524-6529.

11. Borromeo, M.D., et al., ASCL1 and NEUROD1 Reveal Heterogeneity in Pulmonary Neuroendocrine Tumors and Regulate Distinct Genetic Programs. Cell Rep, 2016. 16(5): p. $1259-1272$.

12. Poirier, J.T., et al., Selective tropism of Seneca Valley virus for variant subtype small cell lung cancer. J Natl Cancer Inst, 2013. 105(14): p. 1059-65.

13. Schenk, E.L., et al., A Randomized Double-Blind Phase II Study of the Seneca Valley Virus (NTX-010) versus Placebo for Patients with Extensive-Stage SCLC (ES SCLC) Who Were Stable or Responding after at Least Four Cycles of Platinum-Based Chemotherapy: North Central Cancer Treatment Group (Alliance) N0923 Study. J Thorac Oncol, 2020. 15(1): p. 110-119.

14. Donati, B., E. Lorenzini, and A. Ciarrocchi, BRD4 and Cancer: going beyond transcriptional regulation. Mol Cancer, 2018. 17(1): p. 164.

15. Shi, J. and C.R. Vakoc, The mechanisms behind the therapeutic activity of BET bromodomain inhibition. Mol Cell, 2014. 54(5): p. 728-36.

16. Nicodeme, E., et al., Suppression of inflammation by a synthetic histone mimic. Nature, 2010. 468(7327): p. 1119-23.

17. Cochran, A.G., A.R. Conery, and R.J. Sims, Bromodomains: a new target class for drug development. Nature Reviews Drug Discovery, 2019. 18(8): p. 609-628.

18. Chapuy, B., et al., Discovery and characterization of super-enhancer-associated dependencies in diffuse large B cell lymphoma. Cancer Cell, 2013. 24(6): p. 777-90. 
19. Lovén, J., et al., Selective inhibition of tumor oncogenes by disruption of super-enhancers. Cell, 2013. 153(2): p. 320-34.

20. Ireland, A.S., et al., MYC Drives Temporal Evolution of Small Cell Lung Cancer Subtypes by Reprogramming Neuroendocrine Fate. Cancer Cell, 2020. 38(1): p. 60-78.e12.

21. Wang, S., et al., Target analysis by integration of transcriptome and ChIP-seq data with BETA. Nat Protoc, 2013. 8(12): p. 2502-15.

22. Garnett, M.J., et al., Systematic identification of genomic markers of drug sensitivity in cancer cells. Nature, 2012. 483(7391): p. 570-5.

23. Iorio, F., et al., A Landscape of Pharmacogenomic Interactions in Cancer. Cell, 2016. 166(3): p. 740-754.

24. Schoenherr, C.J. and D.J. Anderson, The neuron-restrictive silencer factor (NRSF): $a$ coordinate repressor of multiple neuron-specific genes. Science, 1995. 267(5202): p. 13603.

25. Polley, E., et al., Small Cell Lung Cancer Screen of Oncology Drugs, Investigational Agents, and Gene and microRNA Expression. J Natl Cancer Inst, 2016. 108(10).

26. George, J., et al., Comprehensive genomic profiles of small cell lung cancer. Nature, 2015. 524(7563): p. 47-53.

27. Yin, M., et al., Potent BRD4 inhibitor suppresses cancer cell-macrophage interaction. Nat Commun, 2020. 11(1): p. 1833.

28. Baine, M.K., et al., SCLC Subtypes Defined by ASCL1, NEUROD1, POU2F3, and YAP1: A Comprehensive Immunohistochemical and Histopathologic Characterization. J Thorac Oncol, 2020. 15(12): p. 1823-1835. 
29. Qu, S., et al., Molecular Subtypes of Primary SCLC Tumors and Their Associations With Neuroendocrine and Therapeutic Markers. J Thorac Oncol, 2021.

30. Frese, K.K., K.L. Simpson, and C. Dive, Small cell lung cancer enters the era of precision medicine. Cancer Cell, 2021. 39(3): p. 297-299.

31. Kresse, S.H., et al., LSAMP, a novel candidate tumor suppressor gene in human osteosarcomas, identified by array comparative genomic hybridization. Genes, Chromosomes and Cancer, 2009. 48(8): p. 679-693.

32. Ntougkos, E., et al., The IgLON Family in Epithelial Ovarian Cancer: Expression Profiles and Clinicopathologic Correlates. Clinical Cancer Research, 2005. 11(16): p. 5764-5768.

33. Lenhart, R., et al., Sensitivity of Small Cell Lung Cancer to BET Inhibition Is Mediated by Regulation of ASCL1 Gene Expression. Mol Cancer Ther, 2015. 14(10): p. 2167-74.

34. Wang, X.D., et al., Subtype-specific secretomic characterization of pulmonary neuroendocrine tumor cells. Nat Commun, 2019. 10(1): p. 3201.

35. Mollaoglu, G., et al., MYC Drives Progression of Small Cell Lung Cancer to a Variant Neuroendocrine Subtype with Vulnerability to Aurora Kinase Inhibition. Cancer Cell, 2017. 31(2): p. 270-285.

36. Cong, L. and F. Zhang, Genome Engineering Using CRISPR-Cas 9 System, in Chromosomal Mutagenesis, S.M. Pruett-Miller, Editor. 2015, Springer New York: New York, NY. p. 197-217.

37. CCBR. RNA Seq Gene Expression Pipeline Documentation. 2018; URL https://github.com/CCBR/Pipeliner/wiki/2.-RNA-Seq-Gene-Expression-PipelineDocumentation. 
38. Zhang, W., et al., Small cell lung cancer tumors and preclinical models display heterogeneity of neuroendocrine phenotypes. Transl Lung Cancer Res, 2018. 7(1): p. 3249.

39. Park, Y.-K. and K. Ge, Glucocorticoid Receptor Accelerates, but Is Dispensable for, Adipogenesis. Molecular and Cellular Biology, 2017. 37(2): p. e00260-16.

40. Li, H. and R. Durbin, Fast and accurate short read alignment with Burrows-Wheeler transform. Bioinformatics, 2009. 25(14): p. 1754-60.

41. Zhang, Y., et al., Model-based Analysis of ChIP-Seq (MACS). Genome Biology, 2008. 9(9): p. R137.

42. Heinz, S., et al., Simple combinations of lineage-determining transcription factors prime cis-regulatory elements required for macrophage and B cell identities. Mol Cell, 2010. 38(4): p. 576-89.

43. Oki, S., et al., ChIP-Atlas: a data-mining suite powered by full integration of public ChIPseq data. EMBO Rep, 2018. 19(12).

44. Galli, G.G., et al., YAP Drives Growth by Controlling Transcriptional Pause Release from Dynamic Enhancers. Mol Cell, 2015. 60(2): p. 328-37.

45. Gertz, J., et al., Distinct properties of cell-type-specific and shared transcription factor binding sites. Mol Cell, 2013. 52(1): p. 25-36.

46. Kurppa, K.J., et al., Treatment-Induced Tumor Dormancy through YAP-Mediated Transcriptional Reprogramming of the Apoptotic Pathway. Cancer Cell, 2020. 37(1): p. 104-122.e12. 
47. Song, K.A., et al., Epithelial-to-Mesenchymal Transition Antagonizes Response to Targeted Therapies in Lung Cancer by Suppressing BIM. Clin Cancer Res, 2018. 24(1): p. 197-208.

48. Liao, S., et al., Genetic modifiers of the BRD4-NUT dependency of NUT midline carcinoma uncovers a synergism between BETis and CDK4/6is. Genes Dev, 2018. 32(17-18): p. 11881200.

49. Lerdrup, M., et al., An interactive environment for agile analysis and visualization of ChIPsequencing data. Nature Structural \& Molecular Biology, 2016. 23(4): p. 349-357.

50. Whyte, Warren A., et al., Master Transcription Factors and Mediator Establish SuperEnhancers at Key Cell Identity Genes. Cell, 2013. 153(2): p. 307-319.

51. Gardner, E.E., et al., Chemosensitive Relapse in Small Cell Lung Cancer Proceeds through an EZH2-SLFN11 Axis. Cancer Cell, 2017. 31(2): p. 286-299.

52. Hann, C.L., et al., Therapeutic efficacy of ABT-737, a selective inhibitor of BCL-2, in small cell lung cancer. Cancer Res, 2008. 68(7): p. 2321-8.

53. Schneeberger, V.E., et al., Quantitation of Murine Stroma and Selective Purification of the Human Tumor Component of Patient-Derived Xenografts for Genomic Analysis. PLoS ONE, 2016. 11: p. e0160587. 


\section{ACKNOWLEDGMENT}

This study was supported by the Center for Cancer Research, the Intramural Program of the National Cancer Institute (NCI) (grant ZIA BC011787: Chen), and in part through a FLEX award from NCI (grant ZIA BC011839: Chen and Schrump) and NCI U01 CA231844 (Oliver). The authors thank Drs. Yulong Li, Yan-Jin Liu, Shih-Hsin Hsiao, Kaitlin C. McLoughlin, and Sichuan Xi for their assistance in this study; Drs. Charles Rudin and J.T. Poirier for providing the SCLC PDXs, which were made available through the SCLC Coordinating Center grant NCI U24 CA213274; Dr. Nenghui Wang from Ningbo Wenda Pharma, China for providing NHWD-870. 


\section{SUPPLEMENTAL INFORMATION}

Figure S1. Knockout of NEUROD1 in H446 cells using gRNA-298.

Figure S2. Knockout of NEUROD1 in DMS-273 cells using gRNA-297.

Figure S3. The top six DNA motifs enriched in the genomic sequences of the NEUROD1 peaks in H446 control cells.

Figure S4. Validation of ChIP-seq results at the NEUROD2 gene locus using ChIP-qPCR.

Figure S5. BET bromodomain proteins physically interact with NEUROD1 in H524 cells.

Figure S6. The NEUROD1-loaded and BRD4-loaded superenhancers identified in H446 control and NEUROD1-KO cells.

Figure S7. LSAMP is regulated by both NEUROD1 and BET bromodomain proteins.

Figure S8. Correlation between the $\mathrm{IC}_{50}$ values of NHWD-870 and JQ1 in SCLC lines $(\mathrm{N}=28)$.

Table S1. The list of the SCLC cell lines $(\mathrm{N}=52)$ used in the present study.

Table S2. The list of genes significantly altered in H446 cells after the knockout of NEUROD1.

Table S3. The list of NEUROD1 peaks identified in H446 control cells (cl.10)

Table S4. The list of NEUROD1-target genes identified by BETA.

Table S5. The list of BRD4 peaks overlayed with NEUROD1 peaks in H446 control cells (cl.10).

Table S6. The list of BRD4 peaks separated from NEUROD1 peaks in H446 control cells (cl.10).

Table S7. The list of NEUROD1-target genes in the NEUROD1 gene signature.

Table S8. The list of genes significantly altered by JQ1 treatment ( $24 \mathrm{hrs}, 1 \mu \mathrm{M})$ in H446 control cells (cl.10).

Table S9. The list of genes significantly altered by JQ1 treatment $(24 \mathrm{hrs}, 1 \mu \mathrm{M})$ in $\mathrm{H} 446$ NEUROD1-KO cells (\#297 cl.2-1).

Table S10. The list of genes significantly altered by JQ1 treatment (24 hours at $0.5 \mu \mathrm{M})$ in CORL279 cells. 
Table S11. The list of NEUROD1-loaded superenhancers in H446 control cells (cl.10).

Table S12. The list of BRD4-loaded superenhancers in H446 control cells (cl.10).

Table S13. The list of BRD4-loaded superenhancers in H446 NEUROD1-KO cells (\#297 c1.2-1).

Table S14. Sensitivity (IC50) of SCLC lines (N=52) to JQ1 and NHWD870.

Table S15. The list of target genes included in the custom siRNA library.

Table S16. The siRNA screen results in H446 and COR-L279 cells.

Table S17. The list of genes significantly altered in SCLC PDX LX22 by NHWD-870 (3mg/Kg, daily for 5 days; $n=6$ tumors per group). 